Supporting Information for:

\title{
Improved mechanochemical fabrication of copper(II) oxide nanoparticles with low E-Factor. Efficient catalytic activity for nitroarenes reduction in aqueous medium
}
Aline Lucchesi Schio, ${ }^{\mathrm{a}}$ Márcio Ronaldo Farias Soares, ${ }^{\mathrm{a}}$ Giovanna Machado ${ }^{\mathrm{b}}$ and Thiago Barcellos*a

a Postgraduate Program in Materials Science and Engineering, University of Caxias do Sul, Francisco Getúlio Vargas 1130, Caxias do Sul, RS, 95070-560, Brazil.

${ }^{\mathrm{b}}$ Laboratory of Microscope and Microanalysis, Northeast Center for Strategic Technologies

(CENETE), Av. Prof. Luís Freire, 1-Cidade Universitária, Recife, PE, 50740-540, Brazil.

"E-mail: thiago.barcellos@ucs.br

Number of pages: 33

Number of figures: 25

Number of Tables: 1

\section{Supporting Information Content}

General experimental and data analysis information.

Figure S1. X-ray diffraction (XRD) pattern of CuONPs from sample MCS-3 .............................S4

Figure S2. Rietveld refinement (using GSAS II software) of X-ray diffraction (XRD) pattern of cuonps from sample MCS-3.

Figure S3. Scanning electron microscopy (SEM) micrographs of cuonps prepared in the absence of $\mathrm{NaCl}$. .S5

Figure S4. Transmission electron microscopy (TEM) micrographs of cuonps (sample MCS-3)

$(a, b, c, d)$, and (e) and size distribution based on 120 counts using the four images. .56

Figure S5. Energy dispersive x-ray (EDS) spectrum of the cuonps obtained by mechanochemical synthesis from the conditions established for the MCS-3. ..............................................S7

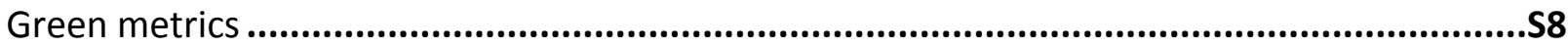

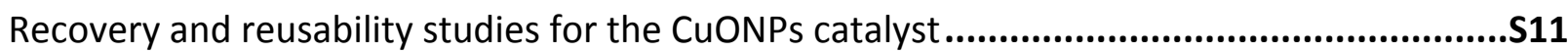

Spectroscopic data for the aminoarenes products.............................................................S12

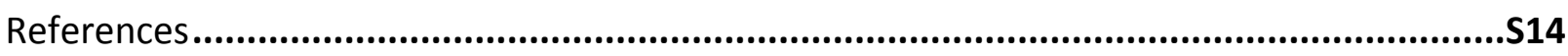

Copies of ${ }^{1} \mathrm{H}$ and ${ }^{13} \mathrm{C} \mathrm{nmr}$ spectra of all aminoarenes products.......................................S16 


\section{General experimental and data analysis information}

The X-ray diffraction (XRD) analyses were obtained on a Shimadzu LabX XRD 6000 diffractometer with a monochromatic $\mathrm{Cu} \mathrm{K \alpha}$ radiation source at $40 \mathrm{kV}, 30 \mathrm{~mA}$ for $\mathrm{Cu} \mathrm{K \alpha}$ $(\lambda=1.5406 \AA)$ with a scan speed of 4 seconds per step from 20 to $75^{\circ}$ at a step size of $0.05^{\circ}$. The indexing was performed with the aid of Using the Crystal Impact Match! Software ${ }^{1}$ (version 3.11.4.199, Evaluation License) and COD-Inorg 2020.12.16 database and GSAS II software (version 4902). ${ }^{2}$ The average crystallite size was determined from the Rietveld refinement method using GSAS II software.

Field emission scanning electron microscopy (FESEM) analyses were performed in a Tescan MIRA3 microscope, operating at $15.0 \mathrm{kV}$, and coupled to energy-dispersive X-ray spectroscopy. The size distribution and morphology of the CuONPs were investigated using an FEI transmission electron microscope (model Morgagni 268D) at an accelerating voltage of 80 $\mathrm{kV}$. In order to perform the TEM analysis, a droplet of a suspension containing CuONPs was ultrasonically dispersed in acetone for $10 \mathrm{~min}$, applied onto a holey carbon grid, and dried. The morphological and particle size distributions were determined from the digital images obtained with a CCD camera. The CuONPs diameter was estimated from ensembles of 100 particles chosen in arbitrary areas of the enlarged micrographs. The diameters of the particles in the micrographs were measured using ImageJ software (version $1.53 \mathrm{e}$ ). ${ }^{3}$ Attenuated total reflection Fourier transform infrared spectroscopy (ATR-FTIR) was read in Perkin Elmer Spectrum 400 equipment in the range of 400-4000 $\mathrm{cm}^{-1}$ using $1 \mathrm{~cm}^{-1}$ resolution. Raman spectroscopy was performed with Raman microscope (InVia, Renishaw) with ion laser beam of $50 \mathrm{~mW}$ power having a wavelength of $532 \mathrm{~nm}$. The thermal stability of CuONPs was examined using a thermogravimetric analyzer (TGA-50, Shimadzu). The sample was heated in the presence of $\mathrm{N}_{2}$ atmosphere in the range of $25-600{ }^{\circ} \mathrm{C}$ with a heating rate of $10{ }^{\circ} \mathrm{C} \mathrm{min}^{-1}$. The UV-Visible spectrum was recorded in a Beckman DU 530 UV visible spectrometry, in the wavelength range of 190-800 $\mathrm{nm}$ at room temperature. For the analysis, it was prepared an aqueous dispersion of the CuONPs (20 ppm), and the absorbance was read in a quartz cuvette. The optical band gap value was determined by plotting the square of optical absorption coefficient as a function of photon energy (Tauc Plot) and by extrapolating the linear region to the energy axis. ${ }^{4}$ The Brunauer-Emmett-Teller (BET) surface area was obtained employing $\mathrm{N}_{2}$ sorption data recorded at $77 \mathrm{~K}$ in a Quantachrome Instruments NOVA 1200e equipment. This analysis was performed in triplicate. The particle size from BET results was calculated by the mathematical relation between the size and surface area of particles, assuming that the particle is spherical, 
where $D$ is the diameter $(\mathrm{nm}), A$ the surface area $\left(\mathrm{m}^{2} \mathrm{~g}^{-1}\right)$ and $\rho$ the density $\left(\mathrm{g} \mathrm{cm}^{-3}\right)$ of the particle (Equation S1). ${ }^{5}$

$$
D=\frac{6000}{A \cdot \rho}
$$

The products from the nitroarenes reductions were identified by gas chromatography-mass spectrometry (GC-MS) in a Hewlett Packard 6890 Series equipment coupled to a mass selective detector model 5973 with HP-INNOWax capillary column $(30 \mathrm{~m}$, id. $0.32 \mathrm{~mm}$ and $0.5 \mu \mathrm{m}$ film thickness) or in a Bruker SCION 456-GC equipment with HP-5 capillary column (60 m, id. $0.32 \mathrm{~mm}$ and $0.5 \mu \mathrm{m}$ film thickness), both using helium as the carrier gas. The NMR spectra were recorded on a Bruker Fourier 300 FT-NMR spectrometer (7.05 Tesla, $300 \mathrm{MHz}$ for the ${ }^{1} \mathrm{H}$ nucleus and $75.48 \mathrm{MHz}$ for the ${ }^{13} \mathrm{C}$ nucleus), running the software TopSpin ${ }^{\mathrm{TM}} 3.2$ for data acquisition and processing. The chemical shifts $(\delta)$ are expressed in part per million (ppm), and the coupling constants are reported in Hz. The spectra were acquired at a temperature of $293 \mathrm{~K}$, using $5 \mathrm{~mm}$ quartz tubes. Melting points were recorded on a Fisatom capillary melting point apparatus, model 431. 


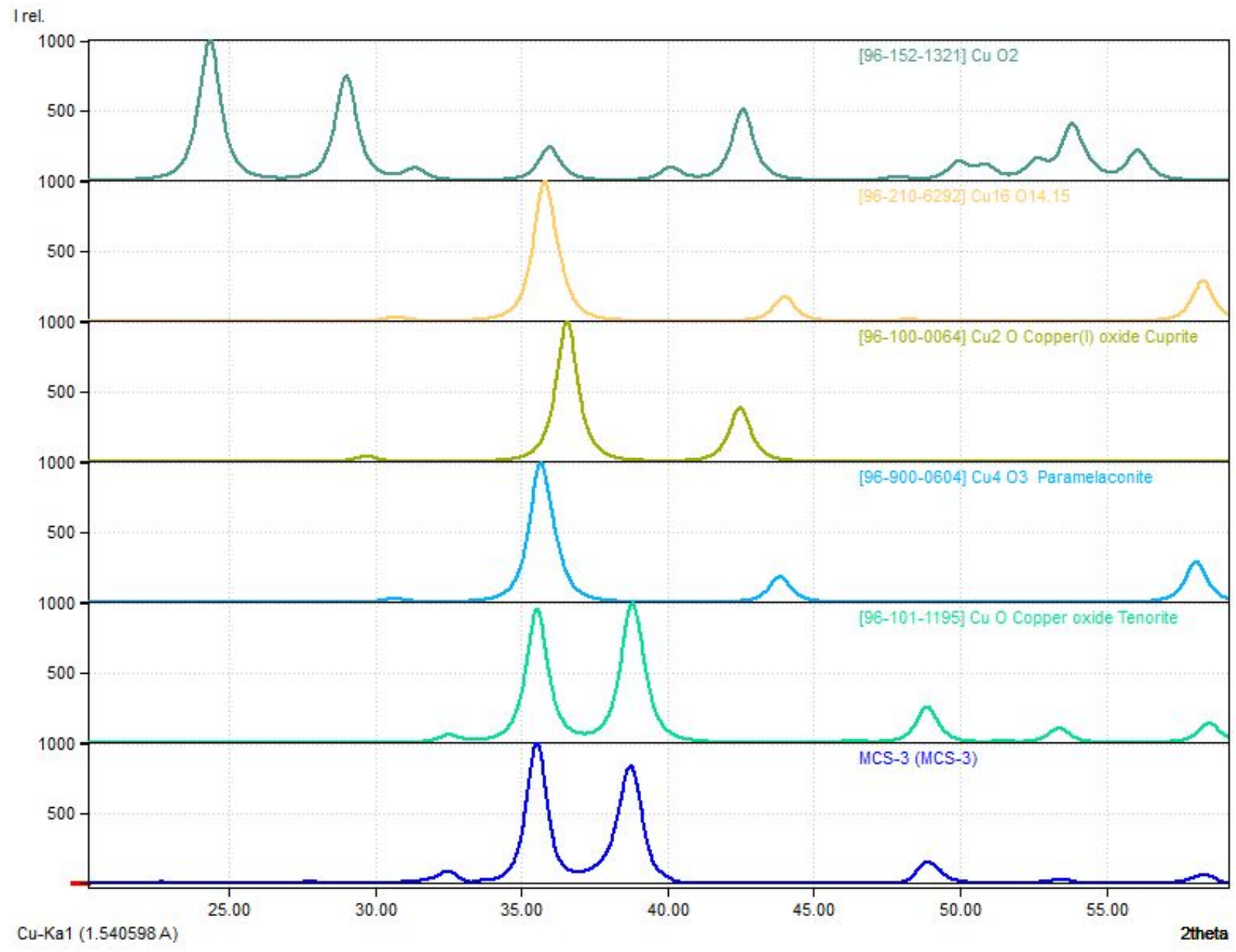

Figure S1. X-ray diffraction (XRD) pattern of CuONPs from sample MCS-3 and the "search-match" routine using Crystal Impact Match! Software (version 3.11.4.199, Evaluation License) and COD-Inorganics 2020.12.16 database. The XRD pattern of sample MCS-3 matches the reference to $\mathrm{CuO}$ tenorite (entry number: 96-101-1195) with a figure-of-merit (FoM) of 0.8175 . 


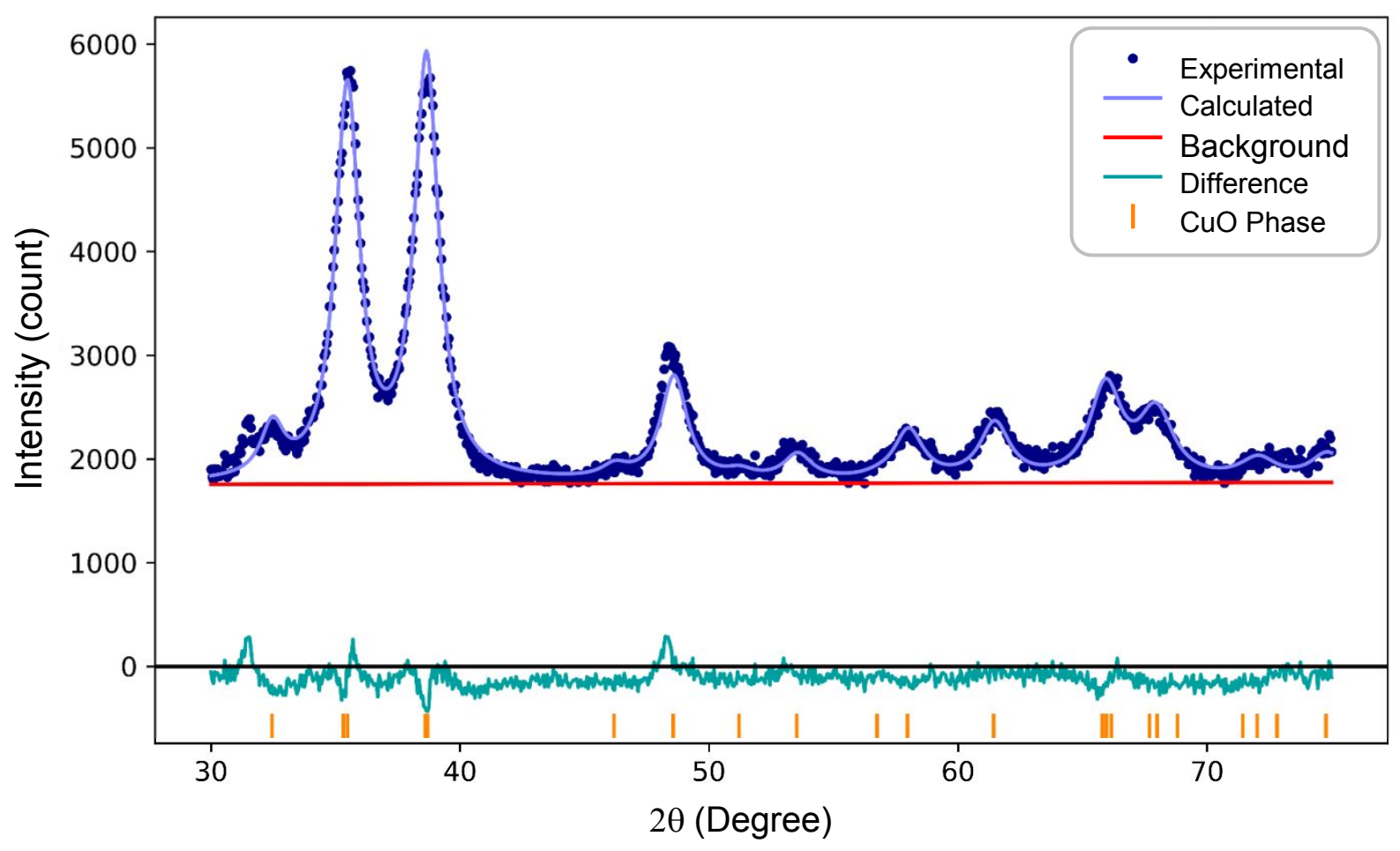

Figure S2. Rietveld refinement (using GSAS II software) of X-ray diffraction (XRD) pattern of CuONPs from sample MSC-3.
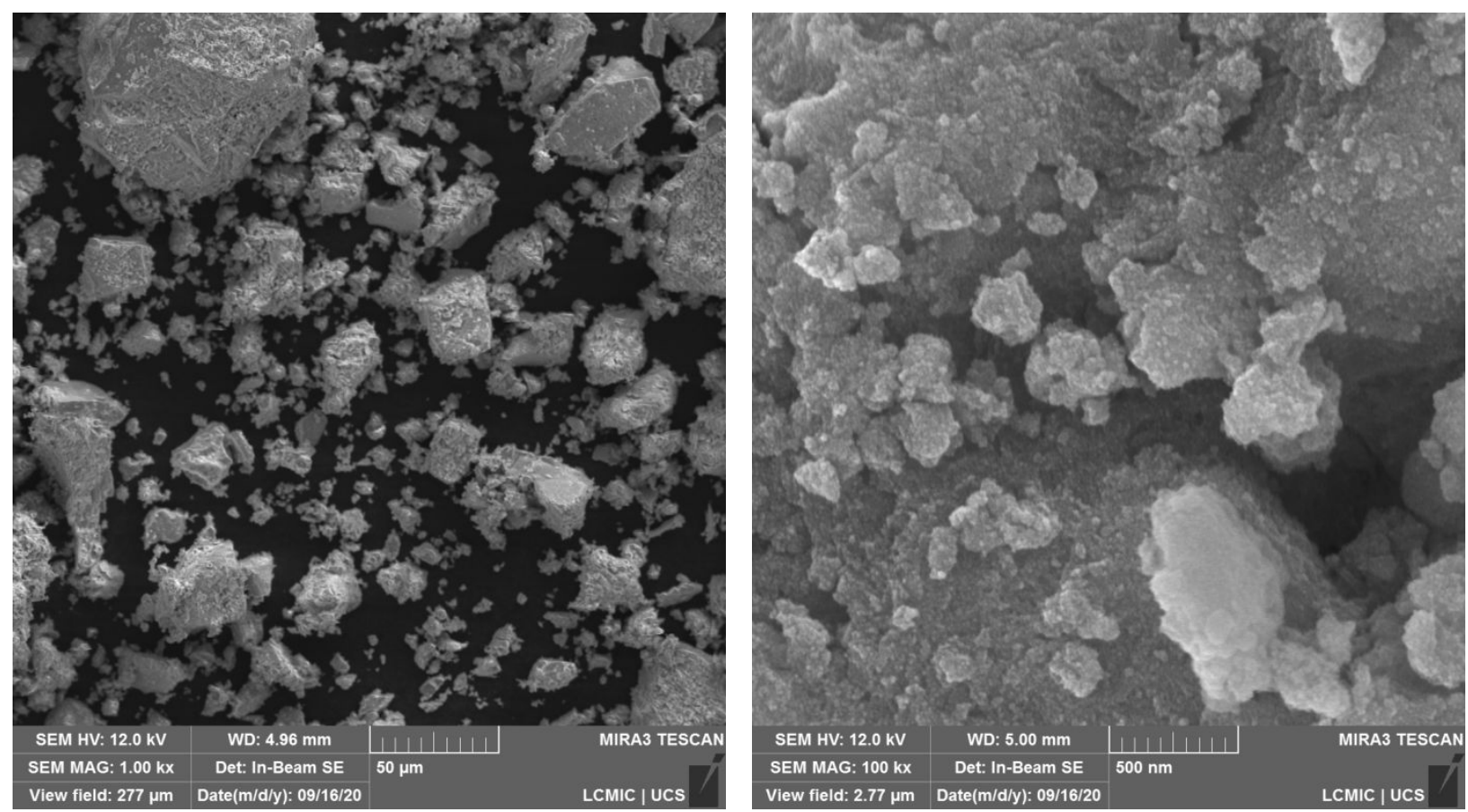

Figure S3. Scanning electron microscopy (SEM) micrographs of CuONPs prepared in the absence of $\mathrm{NaCl}$. 

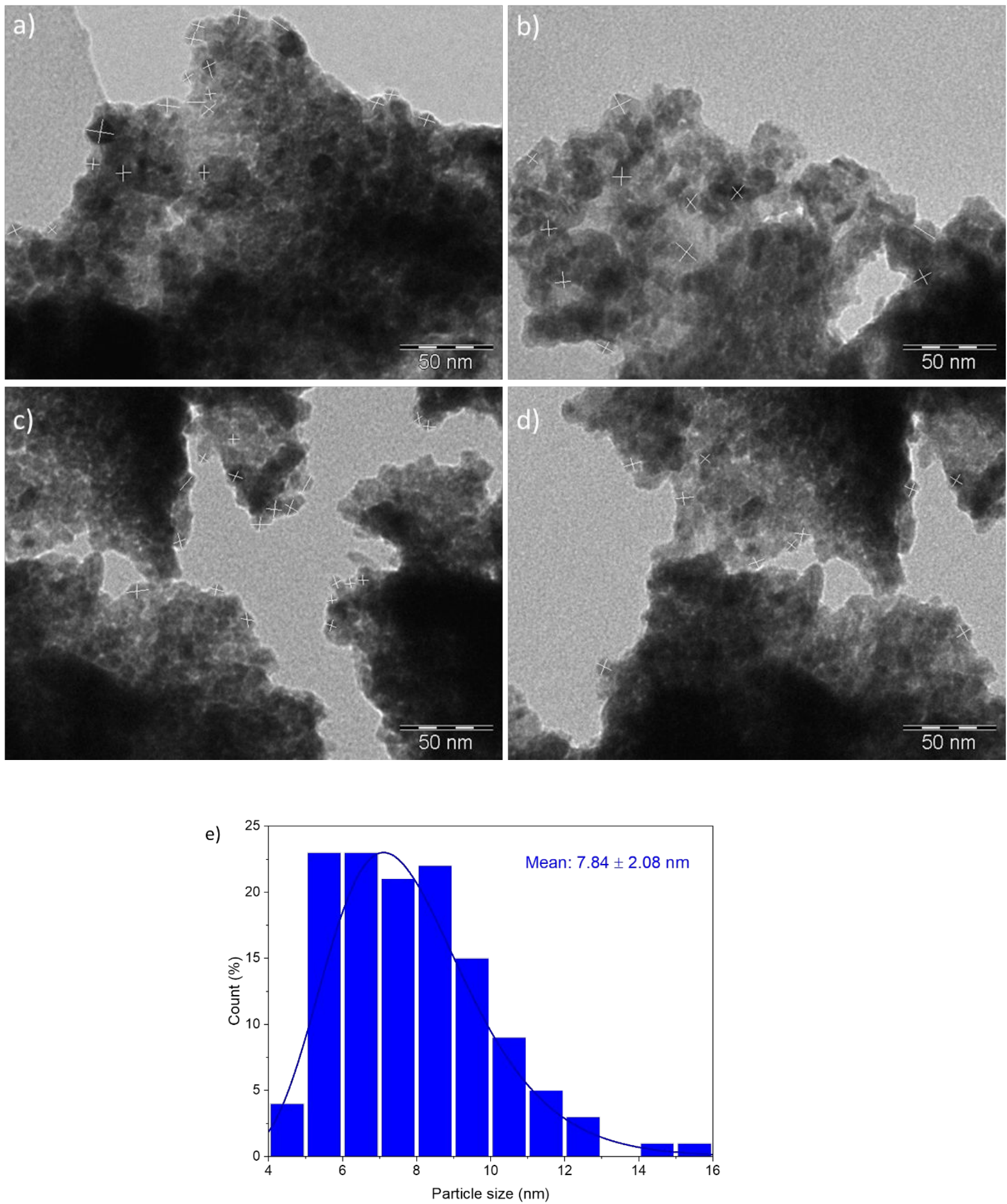

Figure S4. Transmission electron microscopy (TEM) micrographs of CuONPs (sample MCS-3) (a,b,c,d), and (e) and size distribution based on 120 counts using the four images. 


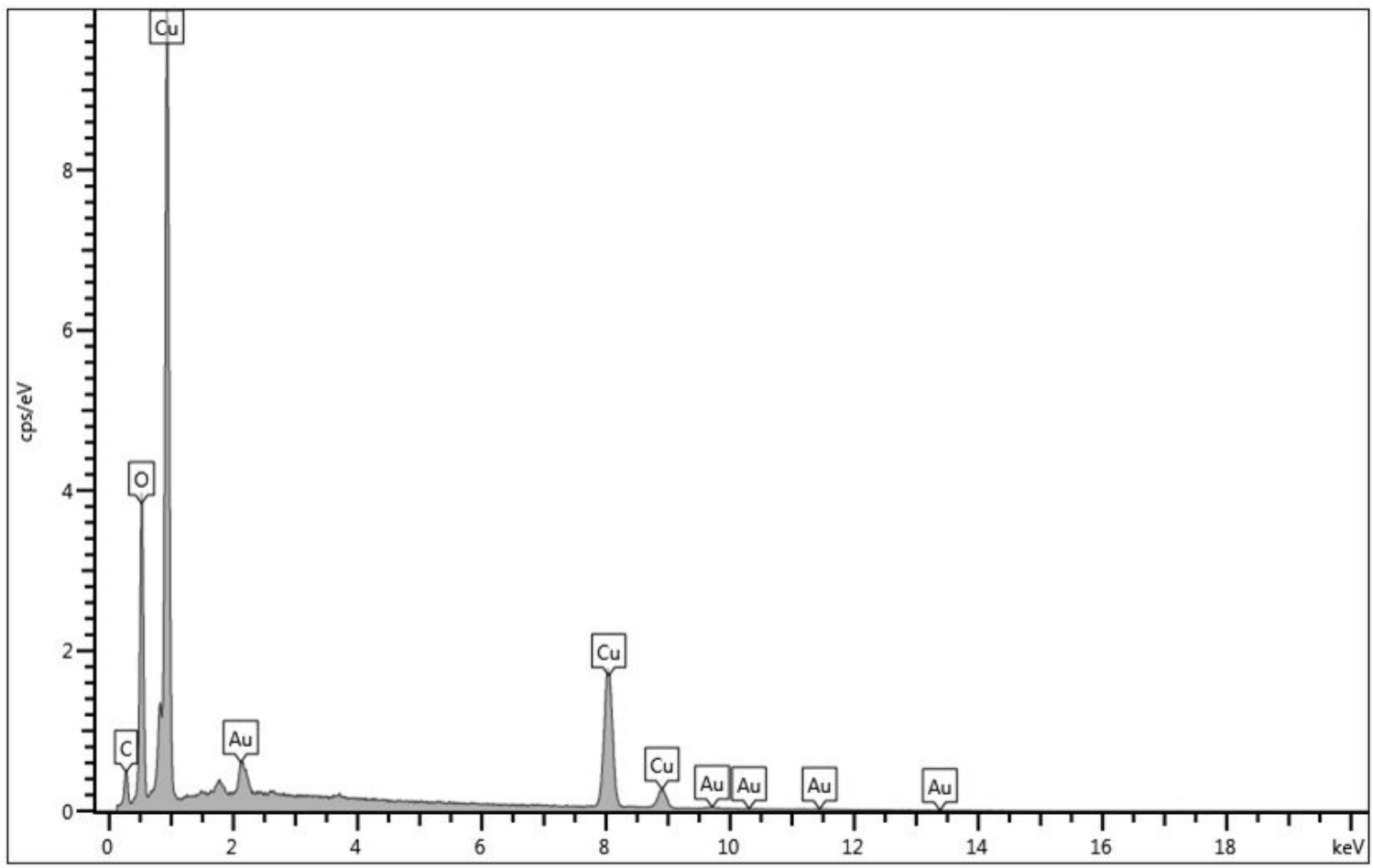

Figure S5. Energy dispersive X-ray (EDS) spectrum of the CuONPs obtained by mechanochemical synthesis from the conditions established for the MCS-3. 


\section{Green Metrics}

Equations for atom economy (Equation S2) and real (or effective) atom economy (Equation S3) measurement. ${ }^{6}$

$$
\begin{gathered}
\mathrm{AE}=\frac{\text { Mol Wt. of desired product }(\mathrm{kg})}{\sum[\text { Mol Wt. of all reagents }(\mathrm{kg})]} \times 100 \% \\
\mathrm{RAE}=\frac{\text { Actual Wt. of desired product }(\mathrm{kg})}{\sum \text { total Wt. of all reagents }(\mathrm{kg})} \\
\text { or } \\
\mathrm{RAE}=\mathrm{AE} \times \text { actual yield }
\end{gathered}
$$

Equations applied for simple E-Factor (sEF) (Equation S4) and complete E-Factor (cEF) (Equation S5). ${ }^{7}$

$$
\begin{gathered}
\mathrm{sEF}=\frac{\sum[\text { total Wt. of all reagents }(\mathrm{kg})]-\text { Actual Wt. of product }(\mathrm{kg})}{\text { Actual Wt. of product }(\mathrm{kg})} \\
\mathrm{cEF}=\frac{\sum[\text { total Wt. of all reagents }(\mathrm{kg})]+\sum[\text { total Wt. of solvent } / \text { water }(\mathrm{kg})]-\text { Actual Wt. of product }(\mathrm{kg})}{\text { Actual Wt. of product }(\mathrm{kg})}
\end{gathered}
$$

\section{E-factor calculations}

The calculated the simple E-factor (sEF) and the complete E-factor (cEF) of the fabrication of the $\mathrm{CuO}$ nanoparticles from the present work and the selected examples are shown in Table S1. The following procedure was adopted: a) all the components (reagents and solvents) reported in the synthetic procedures were considered for the measurements; b) when the weight of the reagents/solvents was not informed, they were calculated from the reported molar or concentration values; c) concerning the weight of the $\mathrm{CuO}$ product, due to no information regarding the isolated yield, it was assumed a $\mathrm{CuO}$ mass corresponding to $99 \%$ yield and calculated based on the mass of the copper precursor.

A representative example of the $\mathrm{sEF}$ and $\mathrm{cEF}$ calculations for the fabrication of $\mathrm{CuONPs}$ of the present work is presented in Figure S6. 
- Simple E-Factor (sEF) calculation for the fabrication of the CuONPs of the present work based on the Equation 4.

a) SEF calculation considering $\mathrm{NaCl}$ and the water consumed in the washing step as a recoverable components.

$\mathrm{sEF}=\frac{\sum[\text { total Wt. of all reagents }(\mathrm{kg})]-\text { Actual Wt. of product }(\mathrm{kg})}{\text { Actual Wt. of product }(\mathrm{kg})}$

$\mathrm{sEF}=\frac{0.00114 \mathrm{~kg}\left[\mathrm{Cu}(\mathrm{OH})_{2}\right]-0.00082 \mathrm{~kg}(\mathrm{CuO})}{0.00082 \mathrm{~kg}(\mathrm{CuO})}=\mathbf{0 . 3 9}$

b) $s E F$ considering $\mathrm{NaCl}$ as a non-recoverable components.

$\mathrm{sEF}=\frac{\sum[\text { total Wt. of all reagents }(\mathrm{kg})]-\text { Actual Wt. of product }(\mathrm{kg})}{\text { Actual Wt. of product }(\mathrm{kg})}$

$\mathrm{sEF}=\frac{0.00114 \mathrm{~kg}\left[\mathrm{Cu}(\mathrm{OH})_{2}\right]+0.00136 \mathrm{~kg}(\mathrm{NaCl})-0.00082 \mathrm{~kg}(\mathrm{CuO})}{0.00082 \mathrm{~kg}(\mathrm{CuO})}=\mathbf{2 . 0 5}$

- Complete E-Factor (cEF) calculation for the fabrication of the CuONPs of the present work based on the Equation 5.

a) CEF calculation considering $\mathrm{NaCl}$ and the water consumed in the washing step as a nonrecoverable components. $\mathrm{cEF}=\frac{\sum[\text { total Wt. of all reagents }(\mathrm{kg})]+\sum[\text { total Wt. of solvent } / \text { water }(\mathrm{kg})]-\text { Actual Wt. of product }(\mathrm{kg})}{\text { Actual Wt. of product }(\mathrm{kg})}$

$\mathrm{cEF}=\frac{0.00114 \mathrm{~kg}\left[\mathrm{Cu}(\mathrm{OH})_{2}\right]+0.00136 \mathrm{~kg}(\mathrm{NaCl})+0.030 \mathrm{~kg}(\text { water })-0.00082 \mathrm{~kg}(\mathrm{CuO})}{0.00082 \mathrm{~kg}(\mathrm{CuO})}=\mathbf{3 8 . 6 3}$

Figure S6. sEF and cEF calculations for the fabrication of CuONPs of the present work. 
Table S1. Simple (sEF) and complete (cEF) Environmental Factor estimated from selected examples in literature for the synthesis of CuONPs.

\begin{tabular}{|c|c|c|c|c|c|c|}
\hline Entry & Method & Reagents/solvents & $\mathrm{CuO}$ weight & $\mathbf{s E F}^{\mathrm{a}}$ & $\mathbf{C E F}^{\mathrm{a}}$ & Reference \\
\hline 1 & $\begin{array}{l}\text { Mechanochemical } \\
\text { synthesis }\end{array}$ & $\begin{array}{l}\text { (a) } \mathrm{Cu}(\mathrm{OH})_{2}(1.14 \mathrm{~g} ; 0.0117 \mathrm{~mol}) \\
\text { (b) } \mathrm{NaCl}(1.36 \mathrm{~g} ; 0.0234 \mathrm{~mol}) \\
\text { (c) water: washing step }(30 \mathrm{~g})\end{array}$ & $\begin{array}{l}0.82 \mathrm{~g}(88 \% \\
\text { yield }) \\
(88 \%, 0.0103 \\
\text { mol })\end{array}$ & $\begin{array}{c}0.39(\mathrm{a}) \\
2.05(\mathrm{a}, \mathrm{b})\end{array}$ & 38.63 & $\begin{array}{l}\text { Present } \\
\text { Work }\end{array}$ \\
\hline 2 & Nanoprecipitation method & $\begin{array}{l}\text { (a) } \mathrm{CuSO}_{4} \cdot 5 \mathrm{H}_{2} \mathrm{O}(0.25 \mathrm{~g} ; 0.001 \mathrm{~mol}) \\
\text { (b) } \mathrm{NaOH}(0.024 \mathrm{~g} ; 0.000003 \mathrm{~mol}) \\
\text { (c) water }(110 \mathrm{~g})\end{array}$ & $\begin{array}{l}0.079 \mathrm{~g} \\
(99 \% \text { yield })^{\mathrm{b}}\end{array}$ & $2.47(\mathrm{a}, \mathrm{b})$ & 1397.43 & [8] \\
\hline 3 & Nanoprecipitation method & $\begin{array}{l}\text { (a) } \mathrm{Cu}\left(\mathrm{CH}_{3} \mathrm{CO}_{2}\right)_{2}(2.7 \mathrm{~g} ; 0.0149 \mathrm{~mol}) \\
\text { (b) Acetic acid }(2.1 \mathrm{~g} ; 0.035 \mathrm{~mol}) \\
\text { (c) ethylene glycol }(710.4 \mathrm{~g} ; 11.45 \mathrm{~mol}) \\
\text { (d) } \mathrm{NaOH}(0.04 \mathrm{~g} ; 0.001 \mathrm{~mol})\end{array}$ & $\begin{array}{l}1.17 \mathrm{~g} \\
(99 \% \text { yield })^{\mathrm{b}}\end{array}$ & $3.13(a, b, d)$ & 609,94 & [9] \\
\hline 4 & $\begin{array}{l}\text { Microwave assisted } \\
\text { nanoprecipitation }\end{array}$ & $\begin{array}{l}\text { (a) } \mathrm{Cu}\left(\mathrm{CH}_{3} \mathrm{CO}_{2}\right)_{2} \cdot \mathrm{H}_{2} \mathrm{O} \quad(0.6 \mathrm{~g} ; 0.003 \\
\text { mol) } \\
\text { (b) } \mathrm{NaOH}(0.00012 \mathrm{~g} ; 0.000003 \mathrm{~mol}) \\
\text { (c) benzyl alcohol }(15.6 \mathrm{~g} ; 15 \mathrm{~mL})\end{array}$ & $\begin{array}{l}0.17(70 \% \\
\text { yield) }\end{array}$ & $2.59(\mathrm{a}, \mathrm{b})$ & 95.97 & {$[10]$} \\
\hline 5 & $\begin{array}{l}\text { Nanoprecipitation method } \\
\text { assisted by capping agent }\end{array}$ & $\begin{array}{l}\text { (a) } \mathrm{CuSO}_{4}(0.319 \mathrm{~g} ; 0.002 \mathrm{~mol}) \\
\text { (b) } \mathrm{NaOH}(0.8 \mathrm{~g} ; 0.02 \mathrm{~mol}) \\
\text { (c) oxalic acidb }(0.36 \mathrm{~g} ; 0.004 \mathrm{~mol}) \\
\text { (d) water }(100 \mathrm{~g})\end{array}$ & $\begin{array}{l}0.16 \mathrm{~g} \\
(99 \% \text { yield })^{\mathrm{b}}\end{array}$ & $8.39(\mathrm{a}, \mathrm{b}, \mathrm{c})$ & 643.28 & [11] \\
\hline 6 & $\begin{array}{l}\text { Nanoprecipitation by } \\
\text { reverse microemulsion } \\
\text { method }\end{array}$ & $\begin{array}{l}\text { (a) } \mathrm{CuSO}_{4} \cdot 5 \mathrm{H}_{2} \mathrm{O}(0.011 \mathrm{~g} ; 0.00005 \mathrm{~mol}) \\
\text { (b) } \mathrm{NaBH}_{4}(0.011 \mathrm{~g} ; 0.0003 \mathrm{~mol}) \\
\text { (c) } \mathrm{TX}-100(3.12 \mathrm{~g} ; 0.005 \mathrm{~mol}) \\
\text { (d) } n \text {-hexanol }(0.49 \mathrm{~g} ; 0.6 \mathrm{~mL}) \\
\text { (e) cyclohexeno }(38.95 \mathrm{~g} ; 50 \mathrm{~mL})\end{array}$ & $\begin{array}{l}0.0035 \mathrm{~g} \\
(99 \% \text { yield })^{\mathrm{b}}\end{array}$ & $5.34(\mathrm{a}, \mathrm{b})$ & $\begin{array}{c}12000.1 \\
2\end{array}$ & {$[12]$} \\
\hline 7 & $\begin{array}{l}\text { Mechanochemical } \\
\text { synthesis of intermediate } \\
\text { followed by calcination } \\
\left(500^{\circ} \mathrm{C}, 2 \mathrm{~h}\right)\end{array}$ & $\begin{array}{l}\text { (a) } \mathrm{Cu}\left(\mathrm{CH}_{3} \mathrm{CO}_{2}\right)_{2} \cdot \mathrm{H}_{2} \mathrm{O} \quad(1.66 \mathrm{~g} ; 0.01 \\
\text { mol) } \\
\text { (b) } \mathrm{CO}\left(\mathrm{NH}_{2}\right)_{2}(0.90 \mathrm{~g} ; 0.015 \mathrm{~mol})\end{array}$ & $\begin{array}{l}0.79 \mathrm{~g} \\
(99 \% \text { yield })^{b}\end{array}$ & $2.26(\mathrm{a}, \mathrm{b})$ & 2.26 & {$[13]$} \\
\hline 8 & $\begin{array}{l}\text { Mechanochemical } \\
\text { synthesis of intermediate } \\
\text { followed by calcination } \\
\left(400^{\circ} \mathrm{C}, 2 \mathrm{~h}\right)\end{array}$ & $\begin{array}{l}\text { (a) } \mathrm{Cu}\left(\mathrm{CH}_{3} \mathrm{CO}_{2}\right)_{2} \cdot \mathrm{H}_{2} \mathrm{O} \quad(1.66 \mathrm{~g} ; 0.01 \\
\text { mol) } \\
\text { (b) }(\mathrm{NH} 4)_{2} \mathrm{C}_{2} \mathrm{O}_{4} \cdot \mathrm{H}_{2} \mathrm{O}(1.42 \mathrm{~g} ; 0.01 \mathrm{~mol})\end{array}$ & $\begin{array}{l}0.79 \mathrm{~g} \\
(99 \% \text { yield })^{\mathrm{b}}\end{array}$ & $2.92(\mathrm{a}, \mathrm{b})$ & 2.92 & {$[14]$} \\
\hline 9 & $\begin{array}{l}\text { Nanoprecipitation method } \\
\text { promoted by plant extract } \\
\text { compounds followed by } \\
\text { calcination }\left(300^{\circ} \mathrm{C}, 2 \mathrm{~h}\right)\end{array}$ & $\begin{array}{l}\text { (a) } \mathrm{CuSO}_{4} \cdot 5 \mathrm{H}_{2} \mathrm{O}(1,25 \mathrm{~g} ; 0.005 \mathrm{~mol}) \\
\text { (b) Plant Extract }(1.5 \mathrm{~g}) \\
\text { (c) water }(115 \mathrm{~mL})\end{array}$ & $\begin{array}{l}0.39 \mathrm{~g} \\
(99 \% \text { yield })^{\mathrm{b}}\end{array}$ & $5.98(a, b)$ & 298.03 & {$[15]$} \\
\hline 10 & $\begin{array}{l}\text { Nanoprecipitation by plant } \\
\text { extract compounds } \\
\text { followed by combustion of } \\
\text { obtained solution at ( } 400 \\
{ }^{\circ} \mathrm{C}, 2 \text { h) }\end{array}$ & $\begin{array}{l}\text { (a) } \mathrm{Cu}\left(\mathrm{NO}_{3}\right)_{2} .3 \mathrm{H}_{2} \mathrm{O}(1.20 \mathrm{~g} ; 0.005 \mathrm{~mol}) \\
\text { (b) Plant Extract }(0.2 \mathrm{~g}) \\
\text { (c) water }(10 \mathrm{~mL})\end{array}$ & $\begin{array}{l}0.39 \mathrm{~g} \\
(99 \% \text { yield })^{\mathrm{b}}\end{array}$ & $2.58(a, b)$ & 28.04 & {$[16]$} \\
\hline 11 & $\begin{array}{l}\text { Nanoprecipitation method } \\
\text { promoted by plant extract } \\
\text { compounds followed by } \\
\text { calcination }\left(450^{\circ} \mathrm{C}, 1 \mathrm{~h}\right)\end{array}$ & $\begin{array}{l}\text { (a) } \mathrm{Cu}\left(\mathrm{NO}_{3}\right)_{2} .3 \mathrm{H}_{2} \mathrm{O}(0.096 \mathrm{~g} ; 0.0004 \\
\text { mol) } \\
\text { (b) Plant Extract }(0.2 \mathrm{~g}) \\
\text { (c) Ammonia }(0,05 \mathrm{~g}) \\
\text { (d) water }(40 \mathrm{~mL})\end{array}$ & $\begin{array}{l}0.031 \\
(99 \% \text { yield })^{b}\end{array}$ & $11.59(\mathrm{a}, \mathrm{b}, \mathrm{c})$ & 1279.77 & {$[17]$} \\
\hline
\end{tabular}

a $\mathrm{sEF}$ and $\mathrm{cEF}$ were determined based on the information described in the respective references and using Equations S3 and S4. The reaction components used for the calculation of the sEF are shown in parentheses and correspond to the reagents informed in the column reagents/solvents. For the calculation of cEF, all components shown in the column reagents/solvents are used. ${ }^{b}$ Due to no information regarding the isolated yield for the uONPs, it was assumed a mass corresponding to $99 \%$ yield and based on the mass of the copper precursor. 


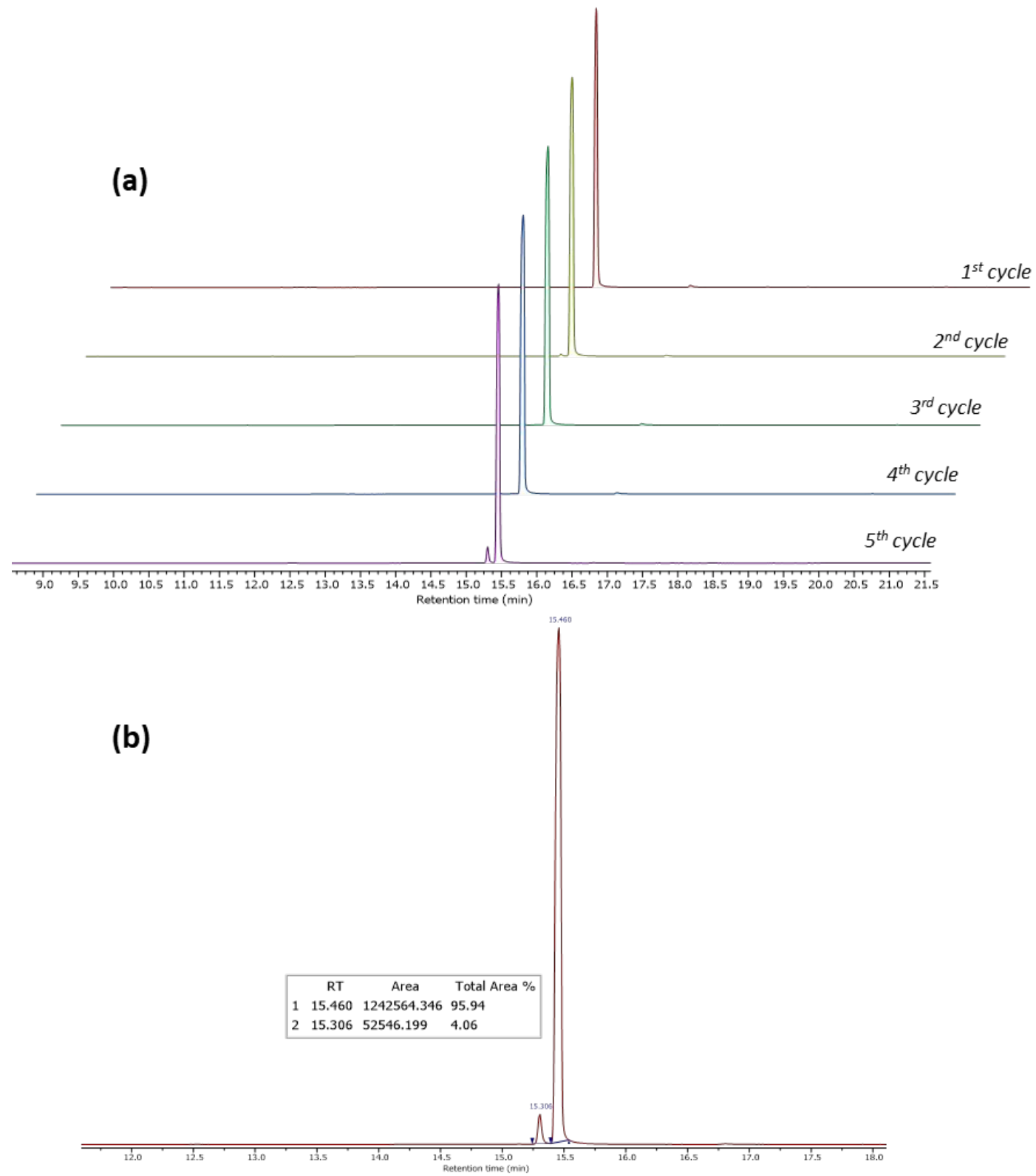

Figure S7. (a) Conversion and selectivity analysis by CG-MS of five consecutive catalyst recycling and (b) chromatogram of the fifth cycle showing $96 \%$ of conversion of nitrobenzene $\left(t_{R}=15.30 \mathrm{~min}\right)$ to aniline $\left(t_{R}=15.46 \mathrm{~min}\right)$. 


\section{Spectroscopic data for the aminoarenes products}<smiles>Nc1ccccc1</smiles>

Aniline (2a): Light yellow liquid obtained from the nitrobenzene reduction (95\% yield). ${ }^{1} \mathrm{H}$ NMR (300 MHz, $\mathrm{CDCl}_{3}$ ): 7.14 (t, $\left.J=7.8 \mathrm{~Hz}, 2 \mathrm{H}\right), 6.75$ (tt, $\left.J=7.5 \mathrm{~Hz}, 1.1 \mathrm{~Hz}, 1 \mathrm{H}\right), 6.66$ (d, $J=7.4 \mathrm{~Hz}, 2 \mathrm{H}$ ), 3.53 (br, 2H) ppm. $\left.{ }^{13} \mathrm{C} \mathrm{NMR} \mathrm{(75} \mathrm{MHz,} \mathrm{CDCl}_{3}\right): \delta 146.5,129.4,118.6,115.2$ ppm. The spectroscopic data matched that previously report. ${ }^{18}$ GC-MS m/z(\%): 93.15 (100\%) [M] $]^{+}, 66.15(53.83 \%)\left[\mathrm{M}-\mathrm{CNH}_{2}\right]^{+}$.<smiles>COc1ccc(N)cc1</smiles>

p-methoxyaniline (2b): Brown solid obtained from the $p$-nitroanisole reduction (98\% yield). Melting point: $58-60{ }^{\circ} \mathrm{C} .{ }^{1} \mathrm{H}$ NMR (300 MHz, DMSO- $\left.d_{6}\right): \delta 3.32$ (br, 2H), $3.74(\mathrm{~s}, 3 \mathrm{H}), 6.63-$ $6.67(\mathrm{~m}, 2 \mathrm{H}), 6.72-6.76(\mathrm{~m}, 2 \mathrm{H}) .{ }^{13} \mathrm{C}$ NMR (75 MHz, DMSO-d $)$ ): $\delta$ 55.7, 114.8, 116.5, 139.9, 152.8. The spectroscopic data matched that previously report ${ }^{19}$

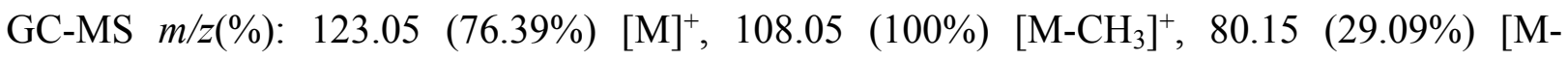
$\left.\mathrm{CH}_{3} \mathrm{OC}\right]^{+}$.<smiles>Nc1ccc(O)cc1</smiles>

p-aminophenol (2c): Reddish yellow solid obtained from the $p$-nitrophenol reduction (96\% yield). Melting point: $185-190{ }^{\circ} \mathrm{C} .{ }^{1} \mathrm{H}$ NMR (300 MHz, DMSO- $\left.d_{6}\right): \delta 8.37$ (br, $\left.1 \mathrm{H}\right), 6.46(\mathrm{~d}, J$ $=8.8,2 \mathrm{H}), 6.40(\mathrm{~d}, J=8.8 \mathrm{~Hz}, 2 \mathrm{H}), 4.37\left(\mathrm{br}, 2 \mathrm{H}, \mathrm{NH}_{2}\right) \cdot{ }^{13} \mathrm{C}$ NMR $\left(75 \mathrm{MHz}, \mathrm{DMSO}-d_{6}\right): \delta$ $148.6,141.1,115.9,115.6$. The spectroscopic data matched that previously report ${ }^{20}$ GC-MS $m / z(\%): 109.15(100 \%)[\mathrm{M}]^{+}, 80.15(38.13 \%)\left[\mathrm{M}-\mathrm{CNH}_{2}\right]^{+}$.<smiles>Nc1cccc(O)c1</smiles>

m-aminophenol (2d): Off-white crystalline solid obtained from the $m$-nitrophenol reduction (97\% yield). Melting point: $120^{\circ} \mathrm{C} .{ }^{1} \mathrm{H}$ NMR (300 MHz, DMSO- $\left.d_{6}\right): \delta 8.81(\mathrm{br}, 1 \mathrm{H}), 6.76(\mathrm{t}, J$ $=8.1 \mathrm{~Hz}, 1 \mathrm{H}), 6.00-5.89(\mathrm{~m}, 3 \mathrm{H}), 4.87(\mathrm{br}, 2 \mathrm{H}) \cdot{ }^{13} \mathrm{C}$ NMR $\left(75 \mathrm{MHz}, \mathrm{DMSO}-d_{6}\right): \delta 101.4$, $103.7,105.8,129.6,150.3,158.5$. The spectroscopic data matched that previously report ${ }^{20}$ GC-MS $m / z(\%): 109.15$ (100\%) [M] $]^{+}, 80.15$ (36.34\%) [M-CNH$]^{+}$. 
<smiles>Nc1ccc(N)cc1</smiles>

p-phenylenediamine (2e): Light violet solid obtained from the $p$-nitroaniline reduction (94\% yield).

Melting point: $142{ }^{\circ} \mathrm{C} .{ }^{1} \mathrm{H}$ NMR $\left(300 \mathrm{MHz}, \mathrm{CDCl}_{3}\right): \delta 6.58(\mathrm{~s}, 4 \mathrm{H}), 3.34$ (brs, $\left.4 \mathrm{H}\right) .{ }^{13} \mathrm{C} \mathrm{NMR}$ $\left(75 \mathrm{MHz}, \mathrm{CDCl}_{3}\right): \delta 116.7,138.6$. The spectroscopic data matched that previously report. ${ }^{20}$ GC-MS $m / z(\%): 108.15(100 \%)[\mathrm{M}]^{+}, 80.15(29.18 \%)\left[\mathrm{M}-\mathrm{CNH}_{2}\right]^{+}$.<smiles>Nc1cccc(N)c1</smiles>

m-phenylenediamine (2f): Off-white solid obtained from the $m$-nitroaniline reduction (95\% yield). Melting point: $64-66{ }^{\circ} \mathrm{C} .{ }^{1} \mathrm{H}$ NMR (300 MHz, DMSO- $\left.d_{6}\right): \delta 6.65(\mathrm{t}, J=7.9 \mathrm{~Hz}, 1 \mathrm{H})$, 5.81-5.76 (m, 3H), 4.64 (brs, 4H). ${ }^{13} \mathrm{C}$ NMR (75 MHz, DMSO- $\left.d_{6}\right): \delta 149.6,129.6,103.5,100.4$. The spectroscopic data matched that previously report. ${ }^{20}$ GC-MS $m / z(\%): 108.15(100 \%)[\mathrm{M}]^{+}, 80.15(54.16 \%)\left[\mathrm{M}-\mathrm{CNH}_{2}\right]^{+}$.<smiles>Nc1cccc(Br)c1</smiles>

$\boldsymbol{m}$-bromoaniline (2g): Yellow-brown liquid obtained from the $m$-bromonitrobenzene reduction (92\% yield). ${ }^{1} \mathrm{H}$ NMR (300 MHz, $\mathrm{CDCl}_{3}$ ): $\delta$ 7.05-6.58 (m, 4H), 3.68 (br, 2H). ${ }^{13} \mathrm{C}$ NMR (75 $\left.\mathrm{MHz}, \mathrm{CDCl}_{3}\right): \delta 147.8,130.6,123.1,121.4,117.8,113.6$. The spectroscopic data matched that previously report. ${ }^{21}$

GC-MS m/z(\%): $171.05(100 \%)[\mathrm{M}]^{+}, 92.15(35.69 \%)[\mathrm{M}-\mathrm{Br}]^{+}$.<smiles>Nc1ccc(C(=O)O)cc1</smiles>

p-aminobenzoic acid (2h): Slight yellow solid obtained from the $p$-nitrobenzoic acid reduction (97\% yied). Melting point: $186-188^{\circ} \mathrm{C} .{ }^{1} \mathrm{H}$ NMR (300 MHz, DMSO- $d_{6}$ ): $\delta 11.96$ (brs, $\left.1 \mathrm{H}\right), 7.60$ $(\mathrm{d}, J=8.8 \mathrm{~Hz}, 2 \mathrm{H}), 6.53(\mathrm{~d}, J=8.7 \mathrm{~Hz}, 2 \mathrm{H}), 5.87$ (brs, $2 \mathrm{H}) .{ }^{13} \mathrm{C}$ NMR $\left(75 \mathrm{MHz}, \mathrm{DMSO}-d_{6}\right): \delta$ $167.9,153.6,131.7,117.3,113.0$. The spectroscopic data matched that previously report. ${ }^{22}$

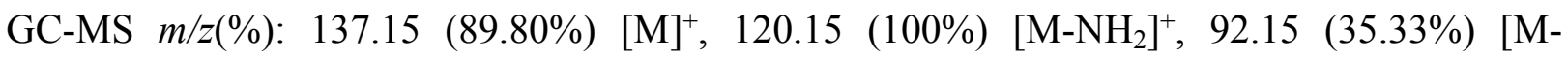
$\left.\mathrm{NH}_{2} \mathrm{CO}\right]^{+}, 65.15(32.50 \%)\left[\mathrm{M}-\mathrm{NH}_{2} \mathrm{COOHC}\right]^{+}$. 
<smiles>Nc1ccccc1CO</smiles>

o-aminobenzyl alcohol (2i): Yellow solid obtained from the $o$-nitrobenzaldehyde reduction (98\% Yield). Melting point: $81-83{ }^{\circ} \mathrm{C} .{ }^{1} \mathrm{H}$ NMR (300 MHz, $\left.\mathrm{CDCl}_{3}\right): \delta 7.16-7.10(\mathrm{~m}, 1 \mathrm{H}), 7.07-$ 7.04 (m, $1 \mathrm{H}), 6.74-6.68$ (m, $2 \mathrm{H}), 4.64$ (s, $2 \mathrm{H}), 3.20$ (br, $3 \mathrm{H}), 4.10$ (br, $2 \mathrm{H}) .{ }^{13} \mathrm{C}$ NMR (75 $\left.\mathrm{MHz}, \mathrm{CDCl}_{3}\right): \delta 146.0,129.4,129.2,124.8,118.2,116.064 .3$. The spectroscopic data matched that previously report. ${ }^{23}$

GC-MS $m / z(\%): 123.15(100 \%)[\mathrm{M}]^{+}, 105.15(79.60 \%)[\mathrm{M}-\mathrm{OH}]^{+}, 78.15(37.42 \%)[\mathrm{M}-$ $\left.\mathrm{COHNH}_{2}\right]^{+}$,

\section{References}

(1) Match! - phase analysis using powder diffraction, crystal impact - dr. H. Putz \& Dr. K. Brandenburg GbR, Kreuzherrenstr. 102, 53227 Bonn, Germany, Https:/Www.Crystalimpact.de/Match.

(2) Toby, B. H.; Von Dreele, R. B. GSAS-II: The genesis of a modern open-source all purpose crystallography software package. J. Appl. Crystallogr. 2013, 46 (2), 544-549, DOI 10.1107/S0021889813003531.

(3) Schneider, C. A.; Rasband, W. S.; Eliceiri, K. W. nih image to imagej: 25 years of image analysis. Nat. Methods 2012, 9 (7), 671-675, DOI 10.1038/nmeth.2089.

(4) Ng, L. K.-S.; Tan, E. J.-C.; Goh, T. W.; Zhao, X.; Chen, Z.; Sum, T. C.; Soo, H. Sen. Mesoporous $\mathrm{SiO}_{2} / \mathrm{BiVO}_{4} / \mathrm{CuO}$ nanospheres for Z-scheme, visible light aerobic $\mathrm{C}-\mathrm{N}$ coupling and dehydrogenation. Appl. Mater. Today 2019, 15, 192-202, DOI 10.1016/j.apmt.2019.01.010.

(5) Ru-Shi, L. Controlled Nanofabrication: Advances and Applications; Jenny Stanford Publishing, 2012, DOI 10.1201/b13155.

(6) Wang, W.; Lü, J.; Zhang, L.; Li, Z. Real atom economy and its application for evaluation the green degree of a process. Front. Chem. Sci. Eng. 2011, 5 (3), 349-354, DOI 10.1007/s11705-010-1030-7.

(7) Sheldon, R. A. The E Factor 25 years on: the rise of green chemistry and sustainability. Green Chem. 2017, 19 (1), 18-43, DOI 10.1039/C6GC02157C.

(8) Chauhan, M.; Sharma, B.; Kumar, R.; Chaudhary, G. R.; Hassan, A. A.; Kumar, S. Green synthesis of $\mathrm{CuO}$ nanomaterials and their proficient use for organic waste removal and antimicrobial application. Environ. Res. 2019, 168, 85-95, DOI 10.1016/j.envres.2018.09.024.

(9) Sahooli, M.; Sabbaghi, S.; Saboori, R. Synthesis and characterization of mono sized CuO nanoparticles. Mater. Lett. 2012, 81, 169-172, DOI 10.1016/j.matlet.2012.04.148.

(10) Nikam, A. V.; Dadwal, A. H. Scalable microwave-assisted continuous flow synthesis of $\mathrm{CuO}$ nanoparticles and their thermal conductivity applications as nanofluids. $A d v$. Powder Technol. 2019, 30 (1), 13-17, DOI 10.1016/j.apt.2018.10.001. 
(11) Bhosale, M. A.; Karekar, S. C.; Bhanage, B. M. Room temperature synthesis of copper oxide nanoparticles: morphological evaluation and their catalytic applications for degradation of dyes and $\mathrm{C}-\mathrm{N}$ bond formation reaction. ChemistrySelect 2016, 1 (19), 6297-6307, DOI 10.1002/slct.201601484.

(12) Kumar, A.; Saxena, A.; De, A.; Shankar, R.; Mozumdar, S. Facile synthesis of sizetunable copper and copper oxide nanoparticles using reverse microemulsions. RSC Adv. 2013, 3 (15), 5015-5021, DOI 10.1039/c3ra23455j.

(13) Tadjarodi, A.; Roshani, R. A green synthesis of copper oxide nanoparticles by mechanochemical method. Curr. Chem. Lett. 2014, 3 (4), 215-220, DOI 10.5267/j.ccl.2014.7.001.

(14) Ameri, B.; Davarani, S. S. H.; Roshani, R.; Moazami, H. R.; Tadjarodi, A. A flexible mechanochemical route for the synthesis of copper oxide nanorods/nanoparticles/nanowires for supercapacitor applications: the effect of morphology on the charge storage ability. J. Alloys Compd. 2017, 695, 114-123, DOI 10.1016/j.jallcom.2016.10.144.

(15) Sreeju, N.; Rufus, A.; Philip, D. Studies on catalytic degradation of organic pollutants and anti-bacterial property using biosynthesized $\mathrm{CuO}$ nanostructures. J. Mol. Liq. 2017, 242 (July 2017), 690-700, DOI 10.1016/j.molliq.2017.07.077.

(16) Udayabhanu; Nethravathi, P. C.; Pavan Kumar, M. A.; Suresh, D.; Lingaraju, K.; Rajanaika, H.; Nagabhushana, H.; Sharma, S. C. Tinospora Cordifolia mediated facile green synthesis of cupric oxide nanoparticles and their photocatalytic, antioxidant and antibacterial properties. Mater. Sci. Semicond. Process. 2015, 33, 81-88, DOI 10.1016/j.mssp.2015.01.034.

(17) Sukumar, S.; Rudrasenan, A.; Padmanabhan Nambiar, D. Green-synthesized rice-shaped copper oxide nanoparticles using Caesalpinia Bonducella seed extract and their applications. ACS Omega 2020, 5 (2), 1040-1051, DOI 10.1021/acsomega.9b02857.

(18) Sharma, M.; Das, B.; Hazarika, A.; Guha, A. K.; Bhargava, S. K.; Bania, K. K. PdO/CuO nanoparticles on zeolite-Y for nitroarene reduction and methanol oxidation. ACS Appl. Nano Mater. 2019, 2 (6), 3769-3779, DOI 10.1021/acsanm.9b00653.

(19) Sharma, S.; Yamini; Das, P. Hydrogenation of nitroarenes to anilines in a flow reactor using polystyrene supported rhodium in a catalyst-cartridge (Cart-Rh@PS). New J. Chem. 2019, 43 (4), 1764-1769, DOI 10.1039/C8NJ04646H.

(20) Portada, T.; Margetić, D.; Štrukil, V. Mechanochemical catalytic transfer hydrogenation of aromatic nitro derivatives. Molecules 2018, 23 (12), 3163, DOI 10.3390/molecules23123163.

(21) Sharma, U.; Kumar, N.; Verma, P. K.; Kumar, V.; Singh, B. Zinc phthalocyanine with PEG-400 as a recyclable catalytic system for selective reduction of aromatic nitro compounds. Green Chem. 2012, 14 (8), 2289-2293, DOI 10.1039/c2gc35452g.

(22) Giri, S.; Das, R.; van der Westhuyzen, C.; Maity, A. An efficient selective reduction of nitroarenes catalyzed by reusable silver-adsorbed waste nanocomposite. Appl. Catal. B Environ. 2017, 209, 669-678, DOI 10.1016/j.apcatb.2017.03.033.

(23) Li, Q.; Zhang, S. Y.; He, G.; Nack, W. A.; Chen, G. Palladium-catalyzed picolinamidedirected acetoxylation of unactivated $\gamma-\mathrm{C}(\mathrm{Sp} 3)-\mathrm{H}$ bonds of alkylamines. Adv. Synth. Catal. 2014, 356 (7), 1544-1548, DOI 10.1002/adsc.201400121. 


\section{Copies of ${ }^{1} \mathrm{H}$ and ${ }^{13} \mathrm{C}$ NMR spectra of all aminoarenes products}
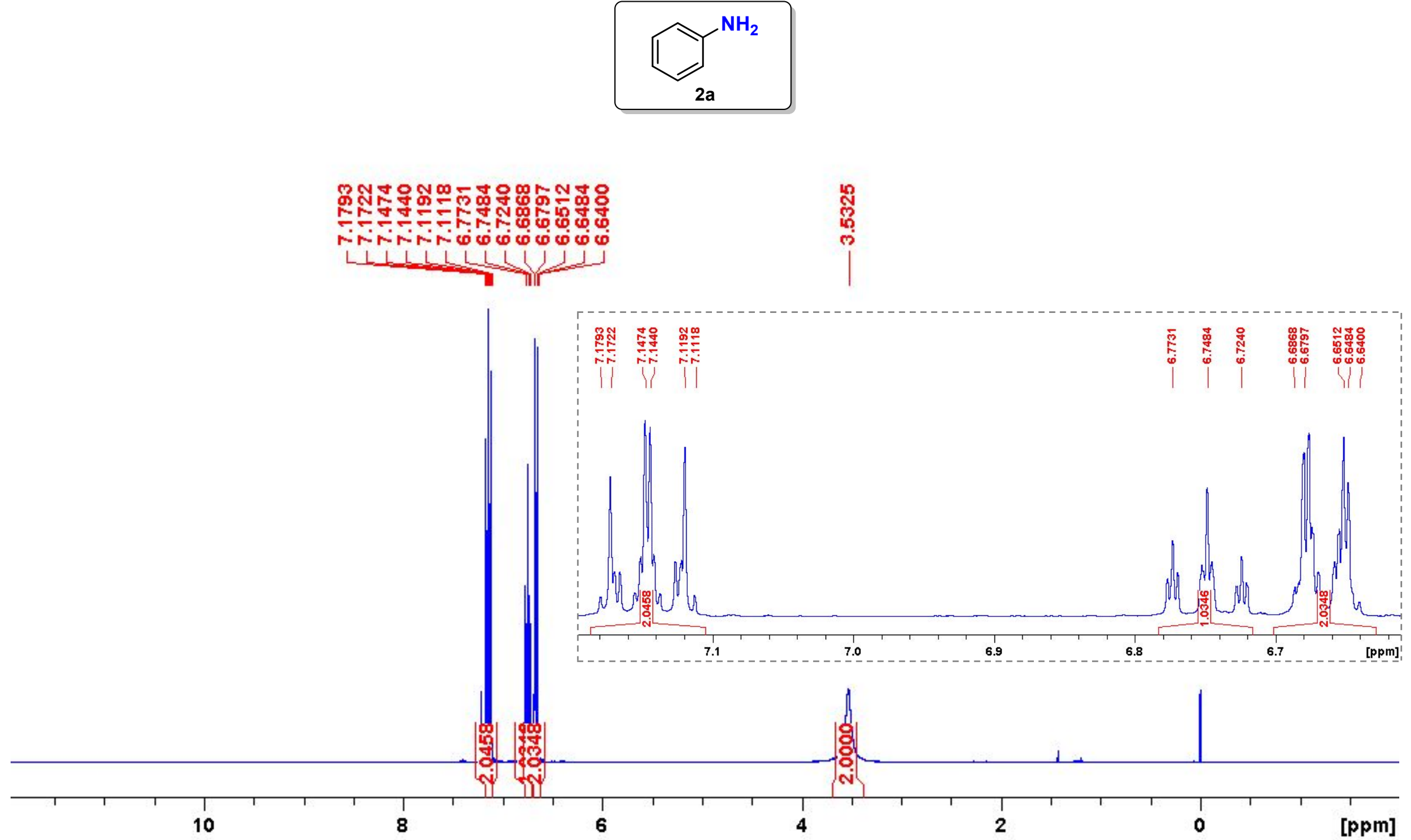

Figure S8. ${ }^{1} \mathrm{H}$ NMR spectrum $\left(300 \mathrm{MHz}, \mathrm{CDCl}_{3}\right)$ of compound $2 \mathbf{a}$. 


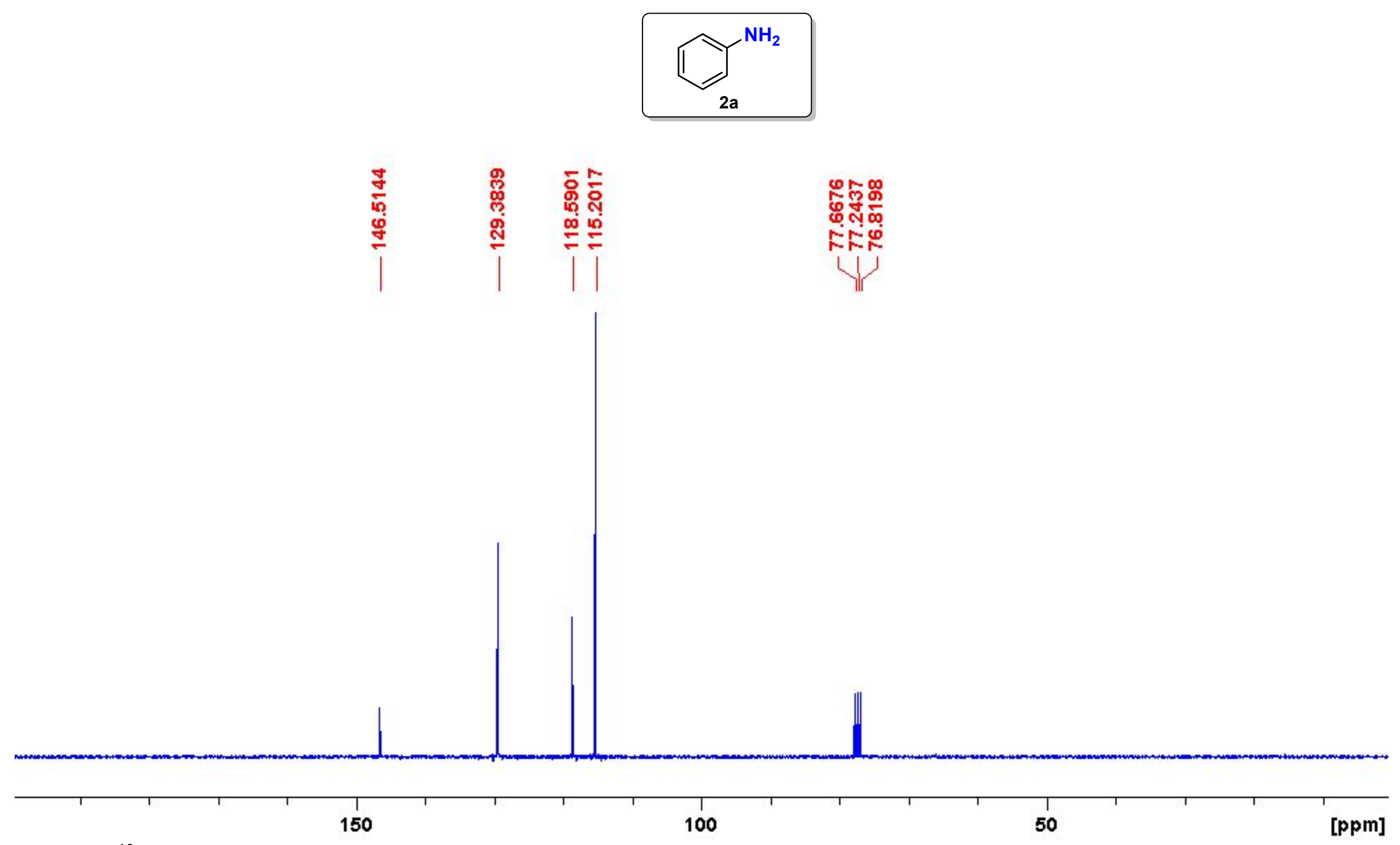

Figure S9. ${ }^{13} \mathrm{C}$ NMR spectrum $\left(75.5 \mathrm{MHz}, \mathrm{CDCl}_{3}\right)$ of compound $2 \mathrm{a}$. 

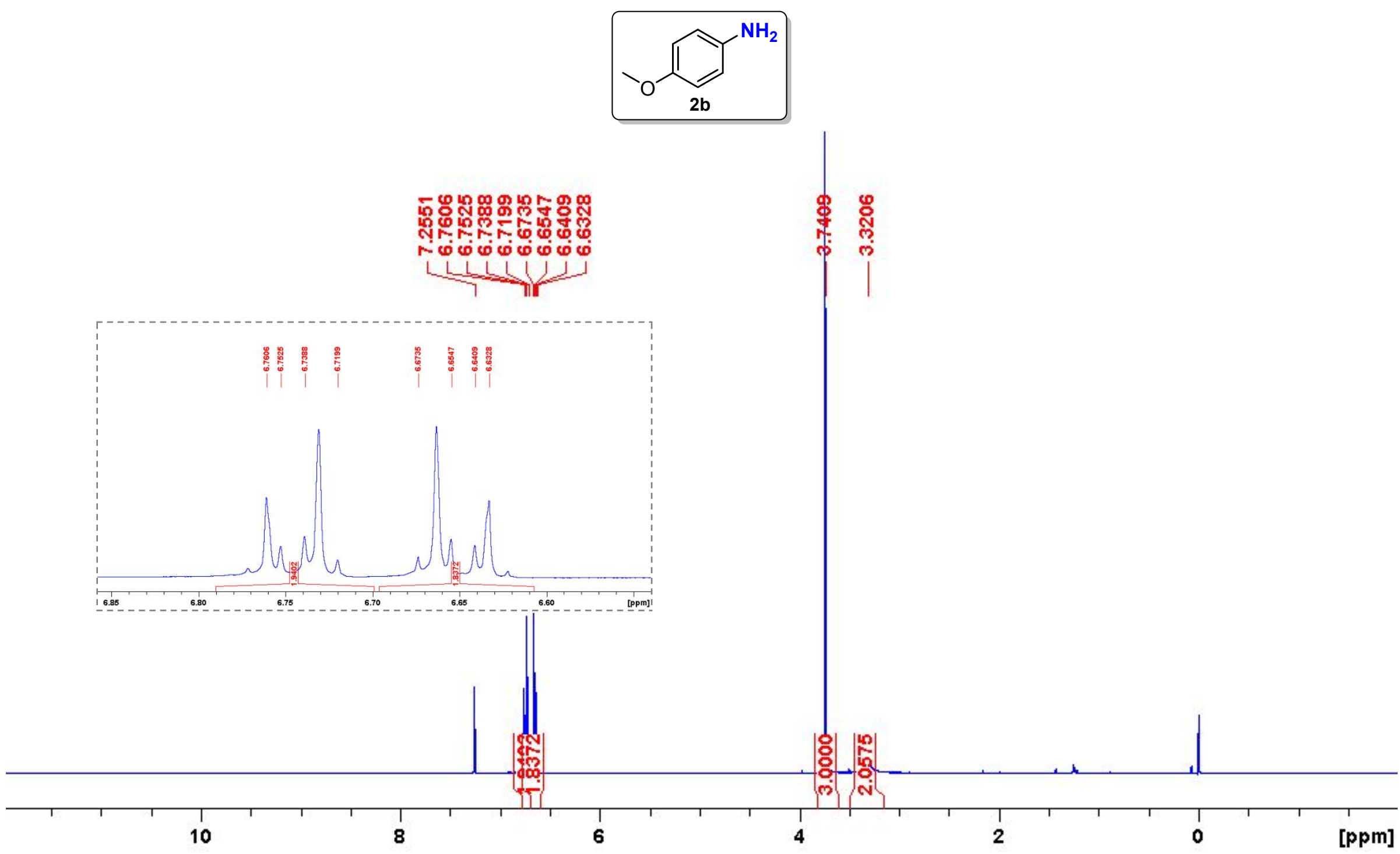

Figure S10. ${ }^{1} \mathrm{H}$ NMR spectrum $\left(300 \mathrm{MHz}, \mathrm{CDCl}_{3}\right)$ of compound $\mathbf{2 b}$. 

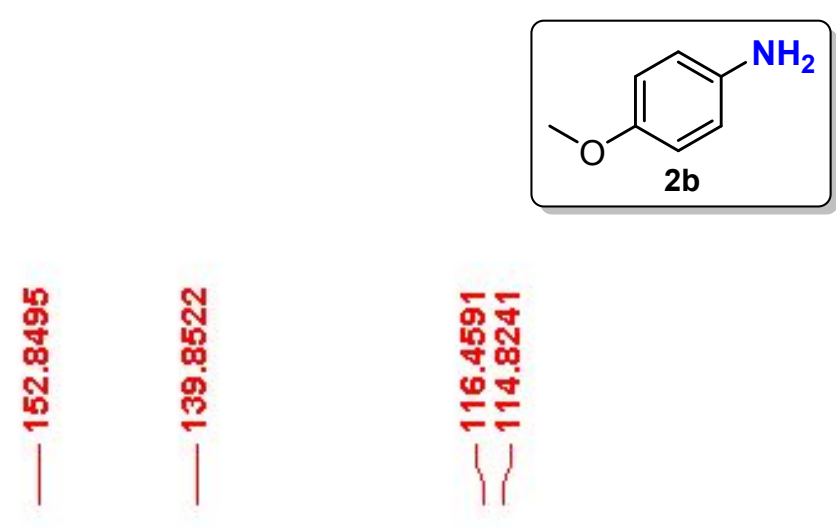

|

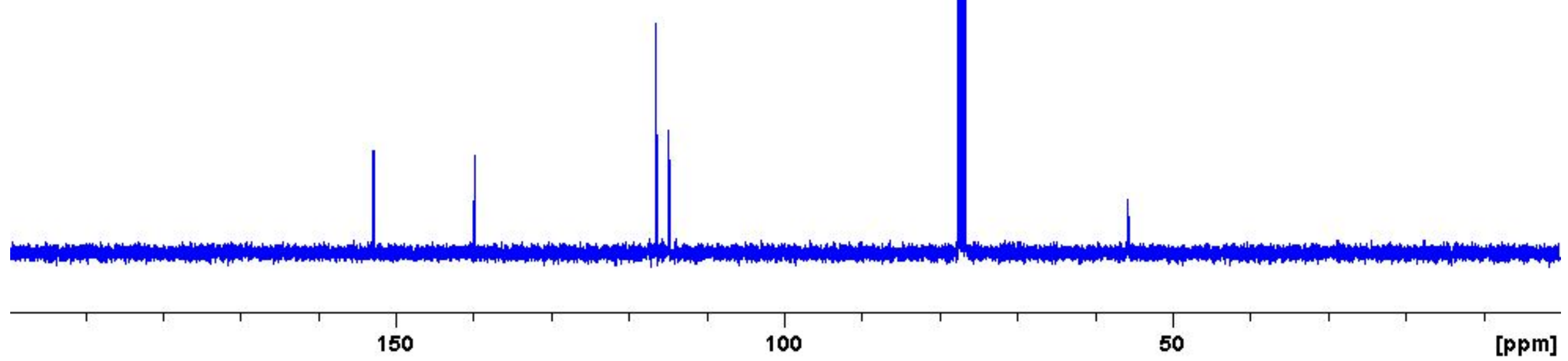

Figure S11. ${ }^{13} \mathrm{C}$ NMR spectrum $\left(75.5 \mathrm{MHz}, \mathrm{CDCl}_{3}\right.$ ) of compound $\mathbf{2 b}$. 

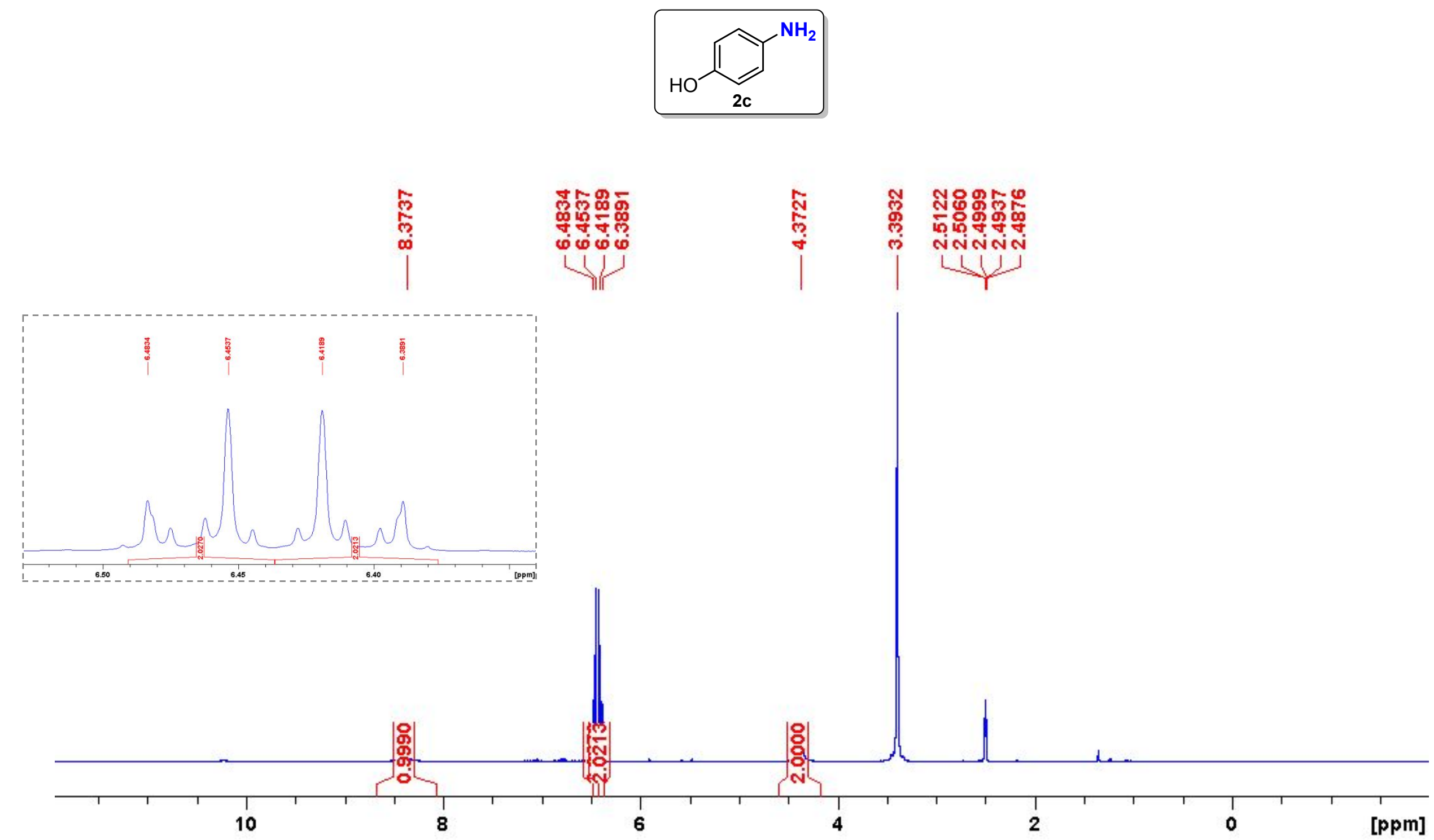

Figure S12. ${ }^{1} \mathrm{H}$ NMR spectrum (300 MHz, DMSO- $d_{6}$ ) of compound 2c. 

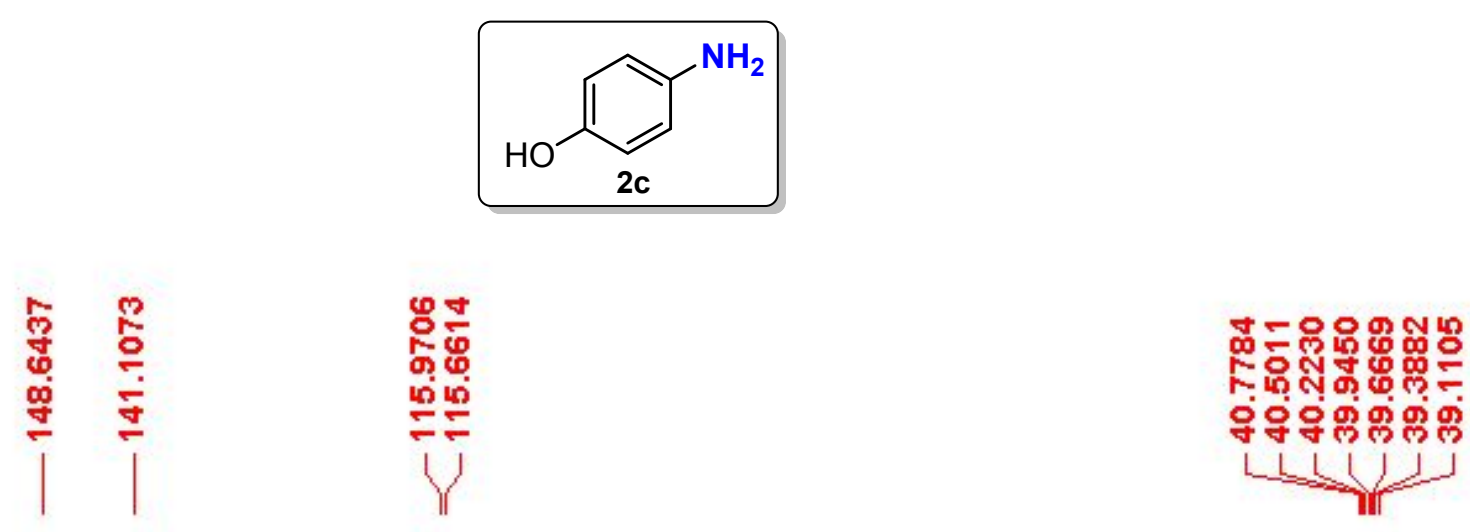

(1)

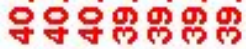
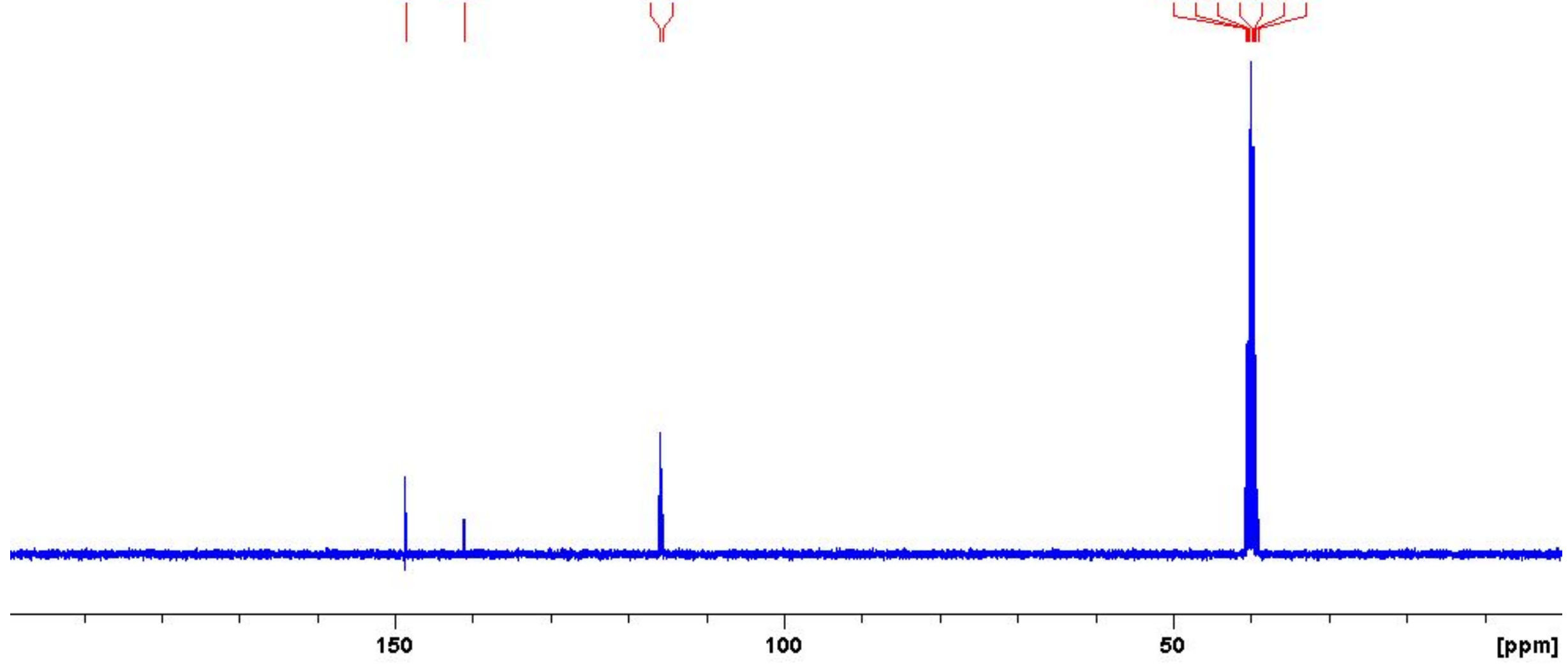

Figure S13. ${ }^{13} \mathrm{C}$ NMR spectrum $\left(75.5 \mathrm{MHz}, \mathrm{DMSO}-d_{6}\right)$ of compound $2 \mathrm{c}$. 

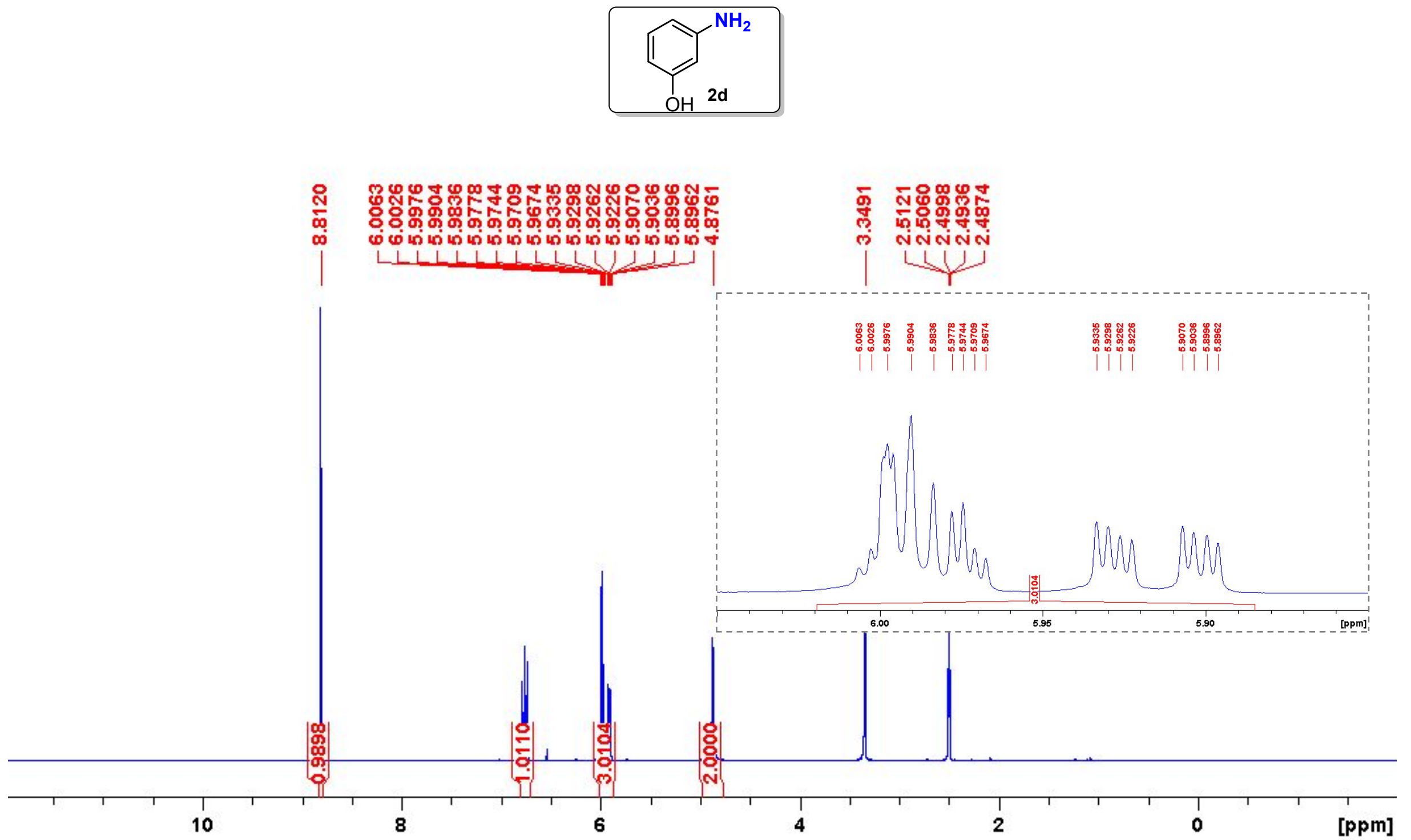

Figure S14. ${ }^{1} \mathrm{H}$ NMR spectrum (300 MHz, DMSO- $d_{6}$ ) of compound 2 d. 

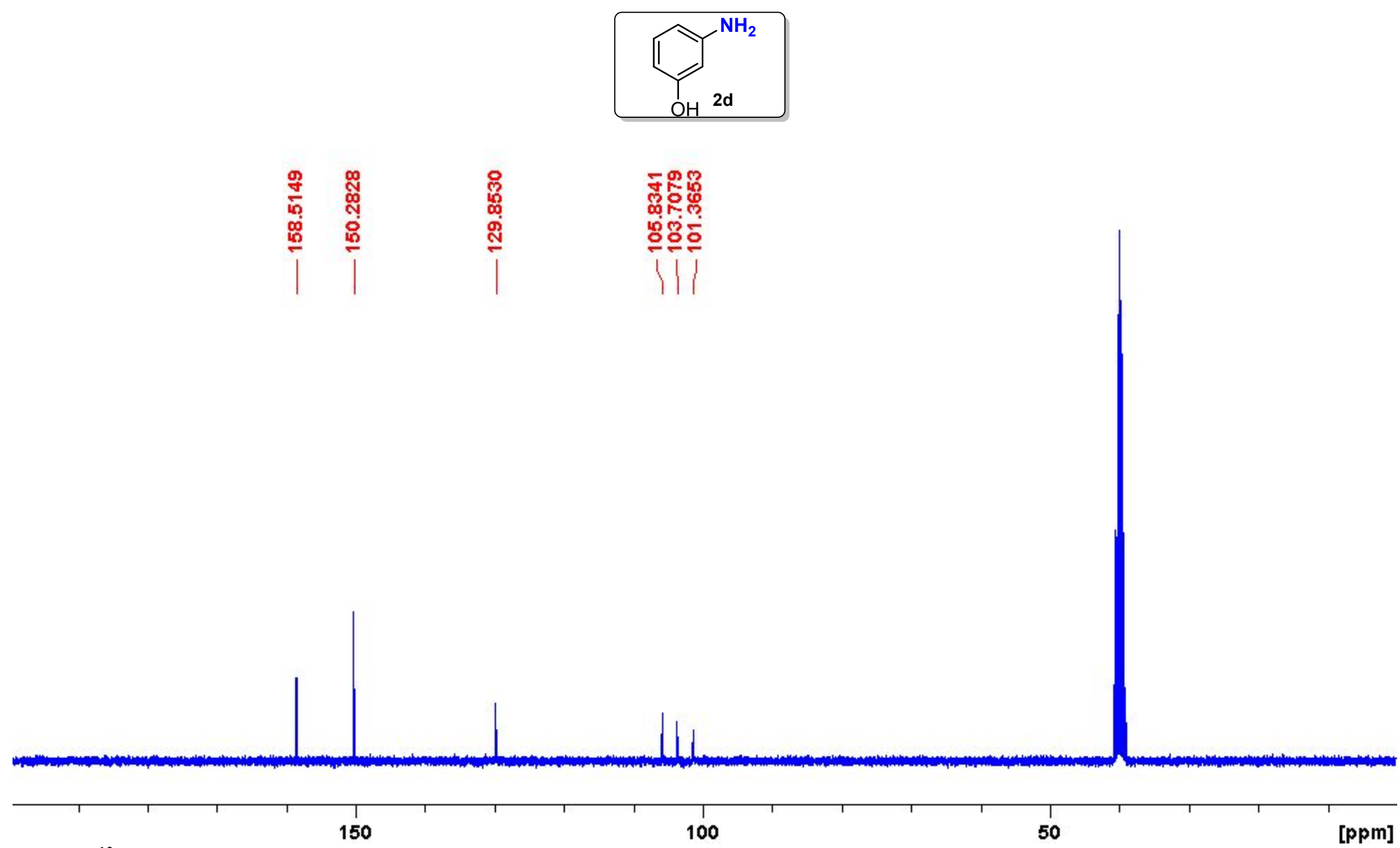

Figure S15. ${ }^{13} \mathrm{C}$ NMR spectrum $\left(75.5 \mathrm{MHz}, \mathrm{DMSO}-d_{6}\right)$ of compound $2 d$. 

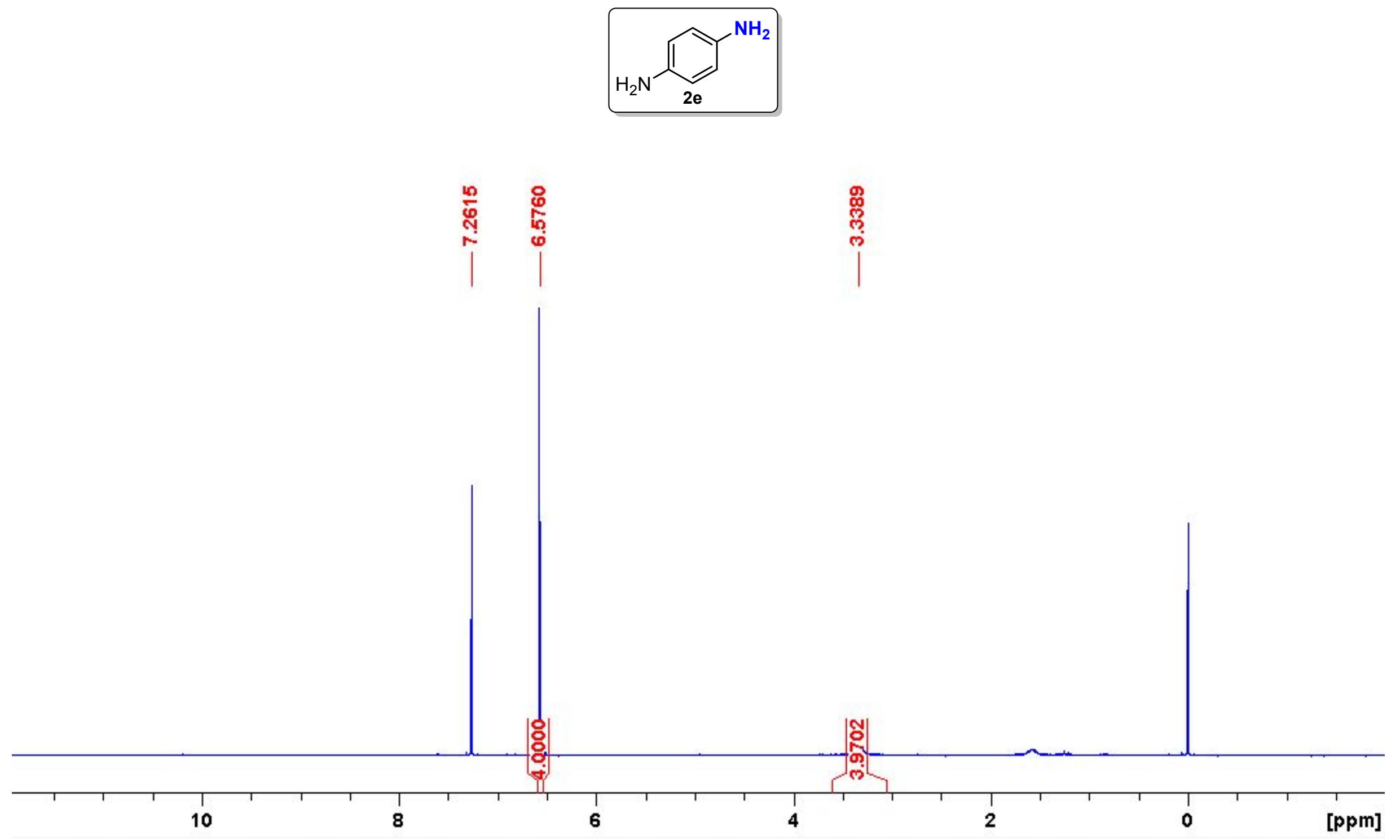

Figure S16. ${ }^{1} \mathrm{H}$ NMR spectrum $\left(300 \mathrm{MHz}, \mathrm{CDCl}_{3}\right)$ of compound $2 \mathbf{e}$. 

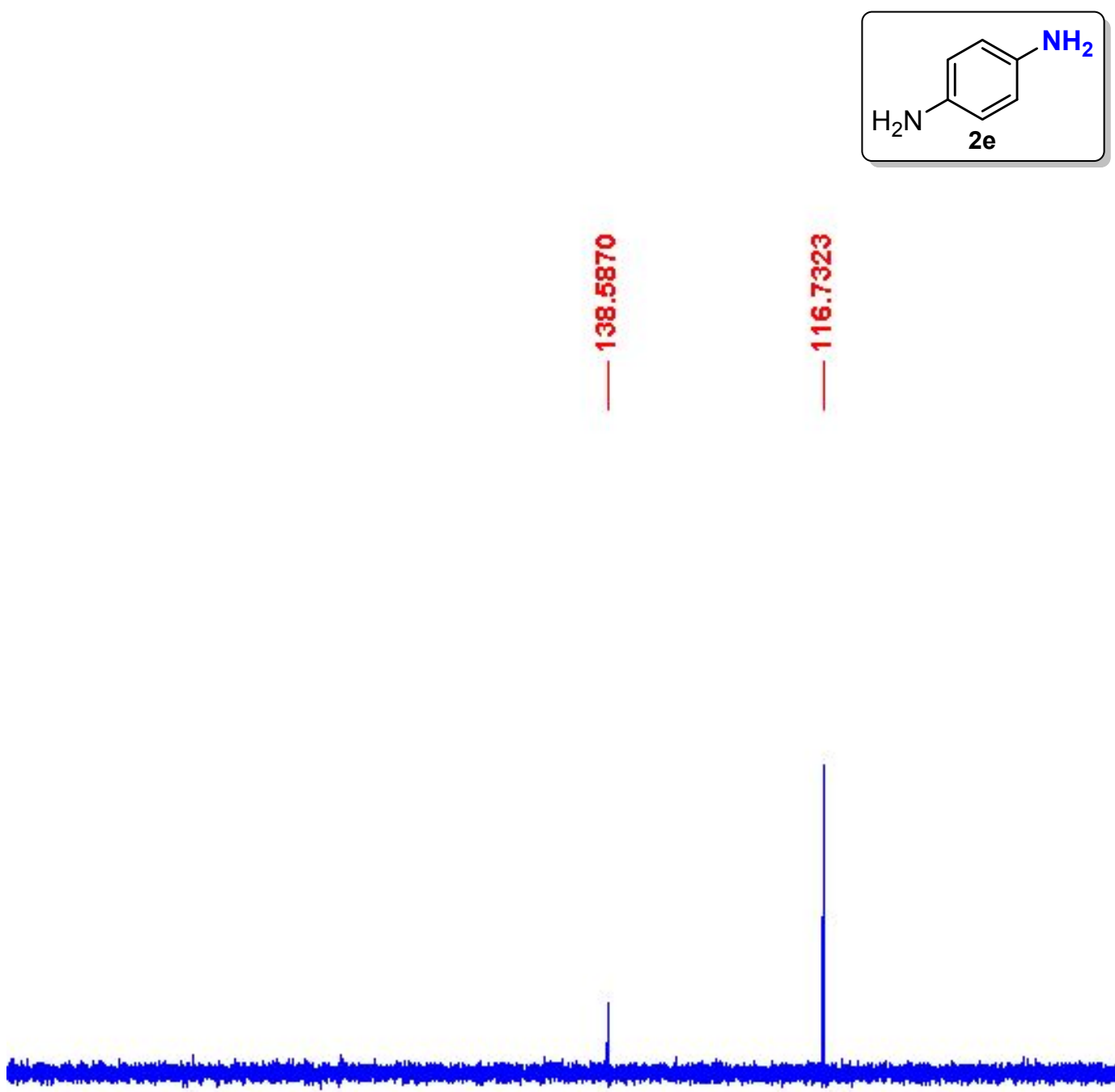

Figure S17. ${ }^{13} \mathrm{C}$ NMR spectrum $\left(75.5 \mathrm{MHz}, \mathrm{CDCl}_{3}\right)$ of compound $2 \mathrm{e}$. 


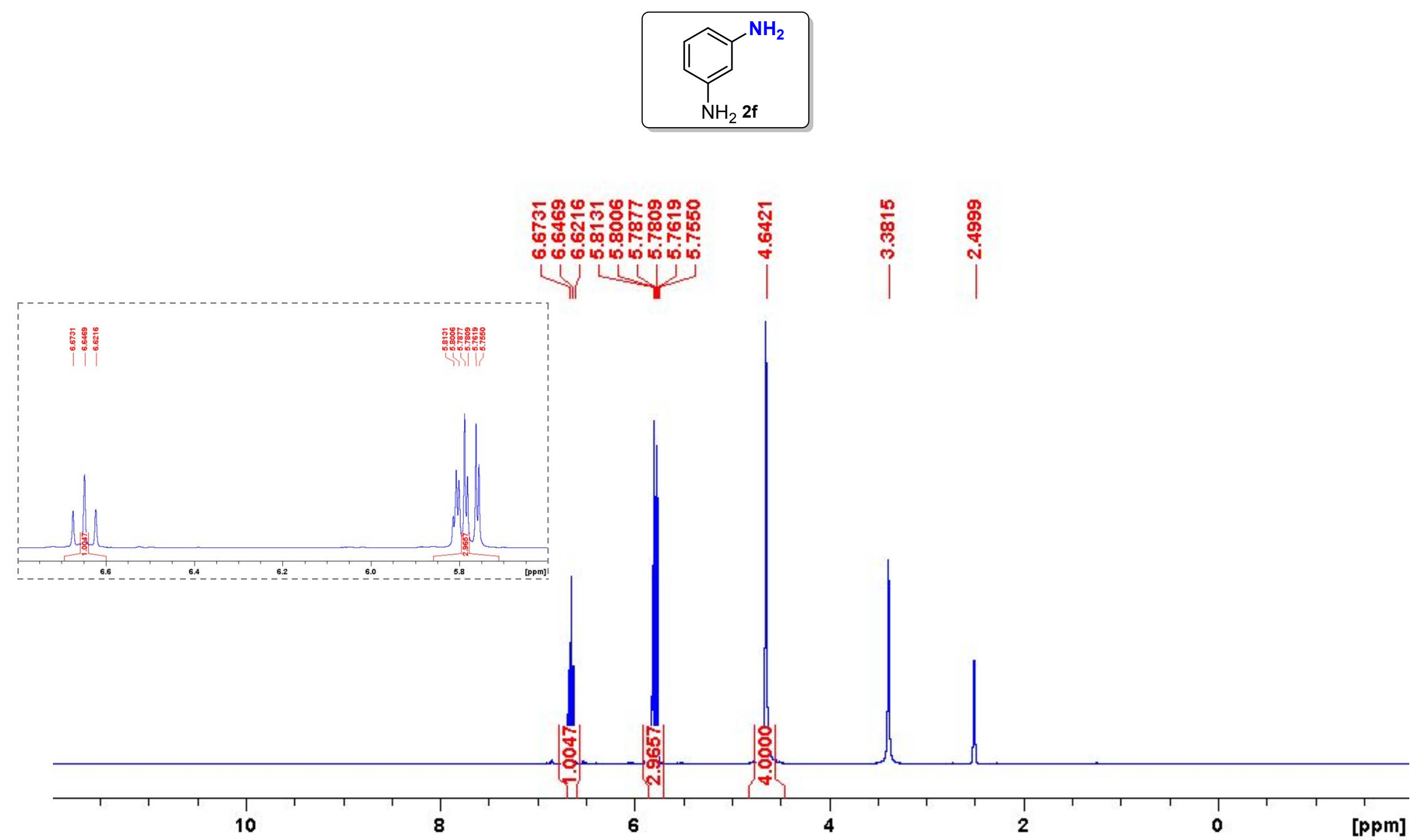

Figure S18. ${ }^{1} \mathrm{H}$ NMR spectrum $\left(300 \mathrm{MHz}\right.$, DMSO- $\left.d_{6}\right)$ of compound $2 \mathbf{f}$. 

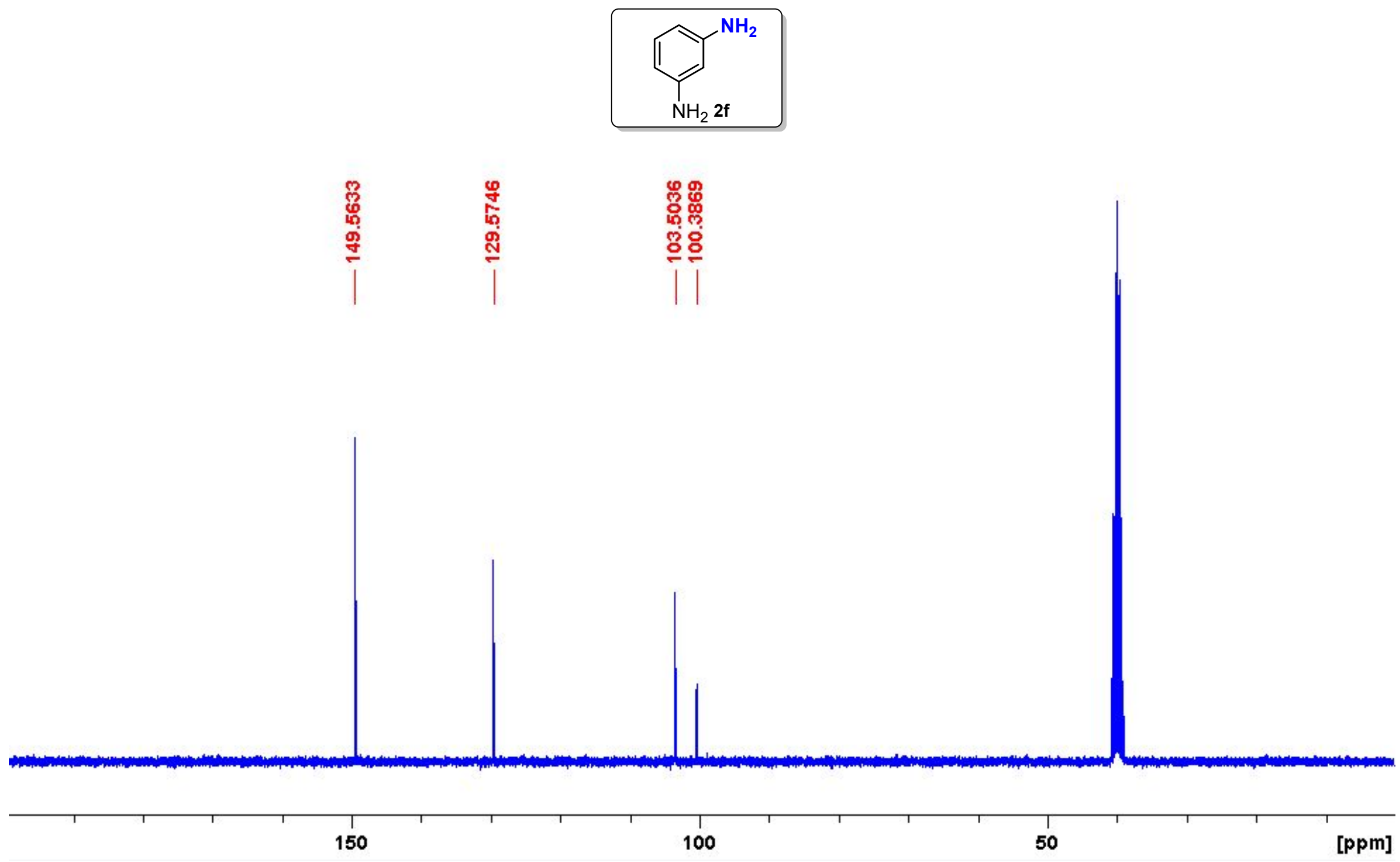

Figure S19. ${ }^{13} \mathrm{C}$ NMR spectrum $\left(75.5 \mathrm{MHz}, \mathrm{DMSO}-d_{6}\right)$ of compound $2 \mathbf{f}$. 

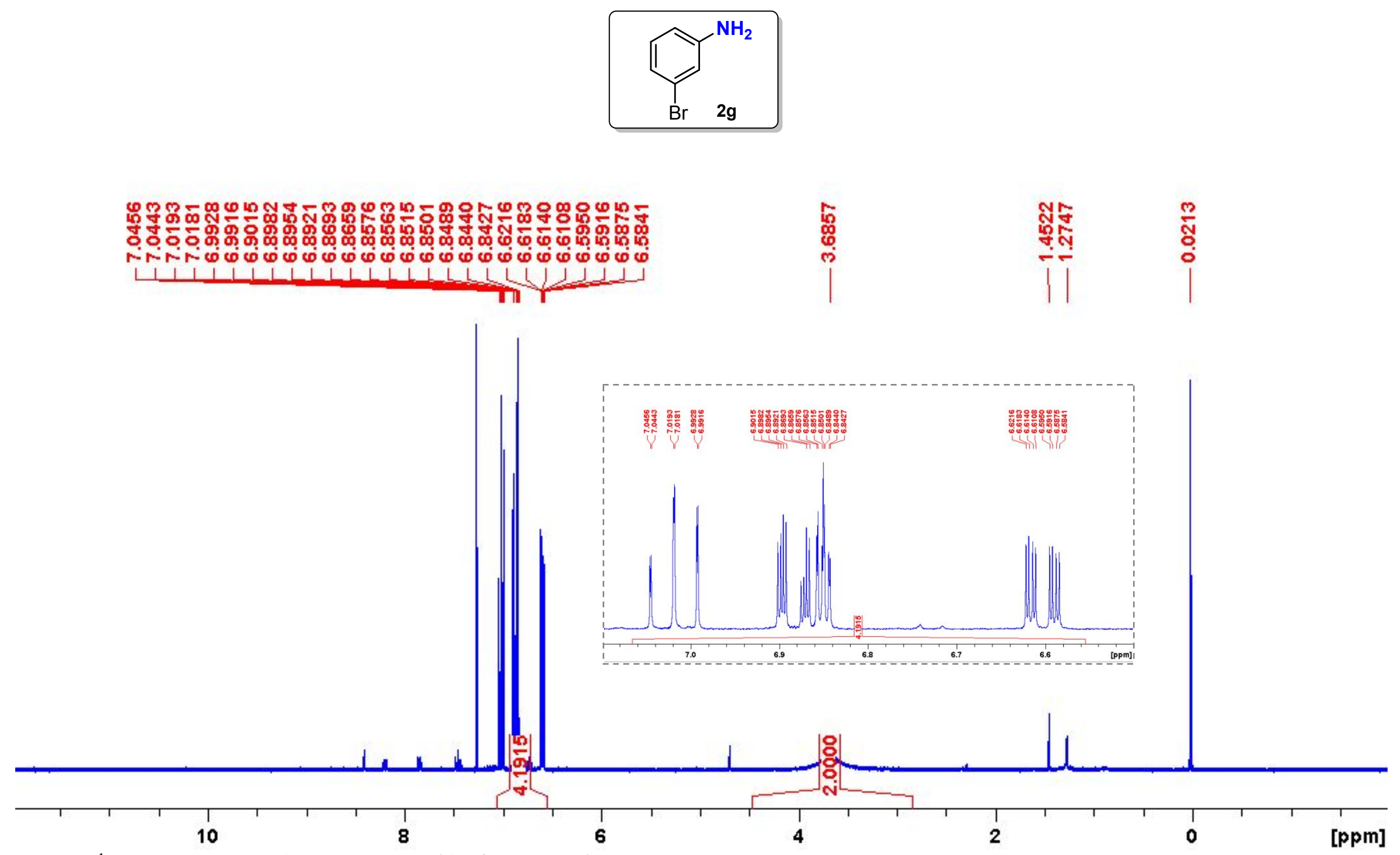

Figure S20. ${ }^{1} \mathrm{H}$ NMR spectrum $\left(300 \mathrm{MHz}, \mathrm{CDCl}_{3}\right)$ of compound $\mathbf{2 g}$. 

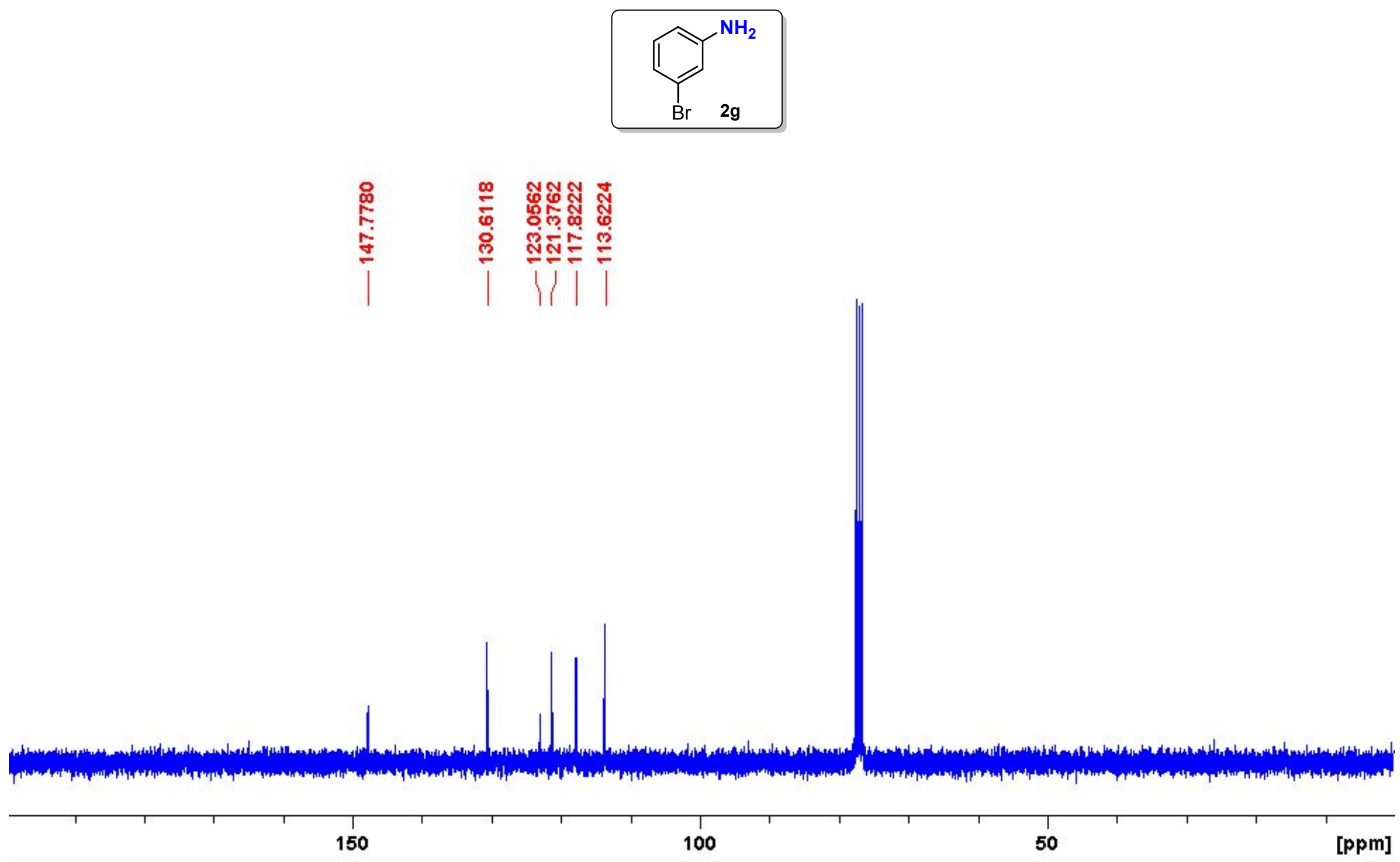

Figure S21. ${ }^{13} \mathrm{C}$ NMR spectrum $\left(75.5 \mathrm{MHz}, \mathrm{CDCl}_{3}\right)$ of compound $2 \mathrm{~g}$. 


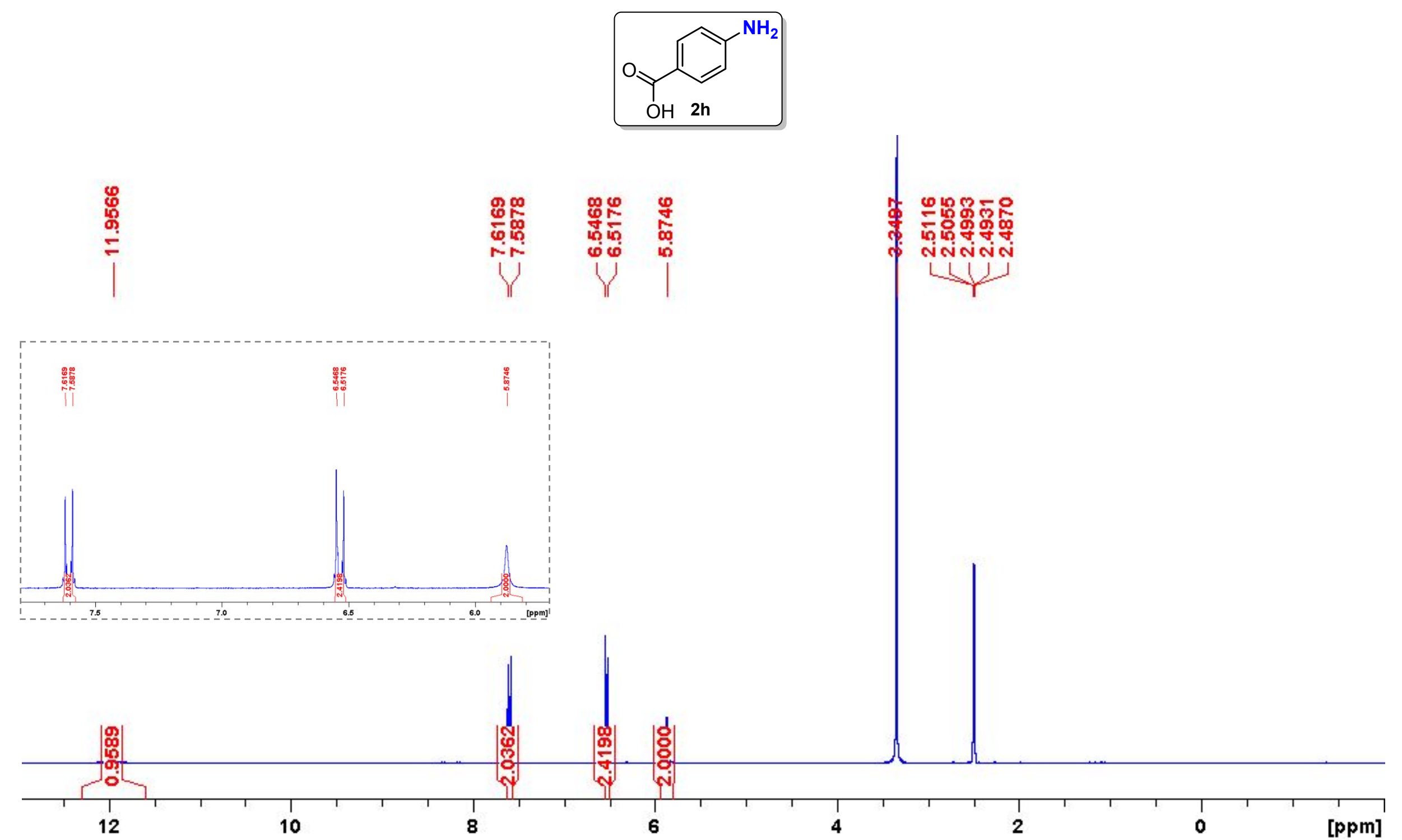

Figure S22. ${ }^{1} \mathrm{H}$ NMR spectrum (300 MHz, DMSO- $d_{6}$ ) of compound $\mathbf{2 h}$. 


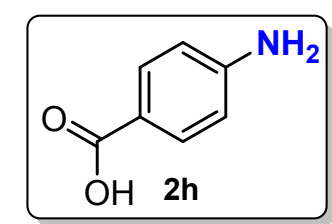

架

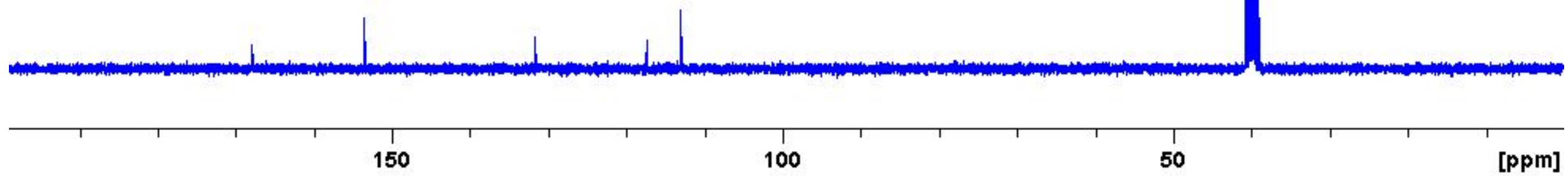

Figure S23. ${ }^{13} \mathrm{C}$ NMR spectrum $\left(75.5 \mathrm{MHz}\right.$, DMSO- $\left.d_{6}\right)$ of compound $\mathbf{2 h}$. 

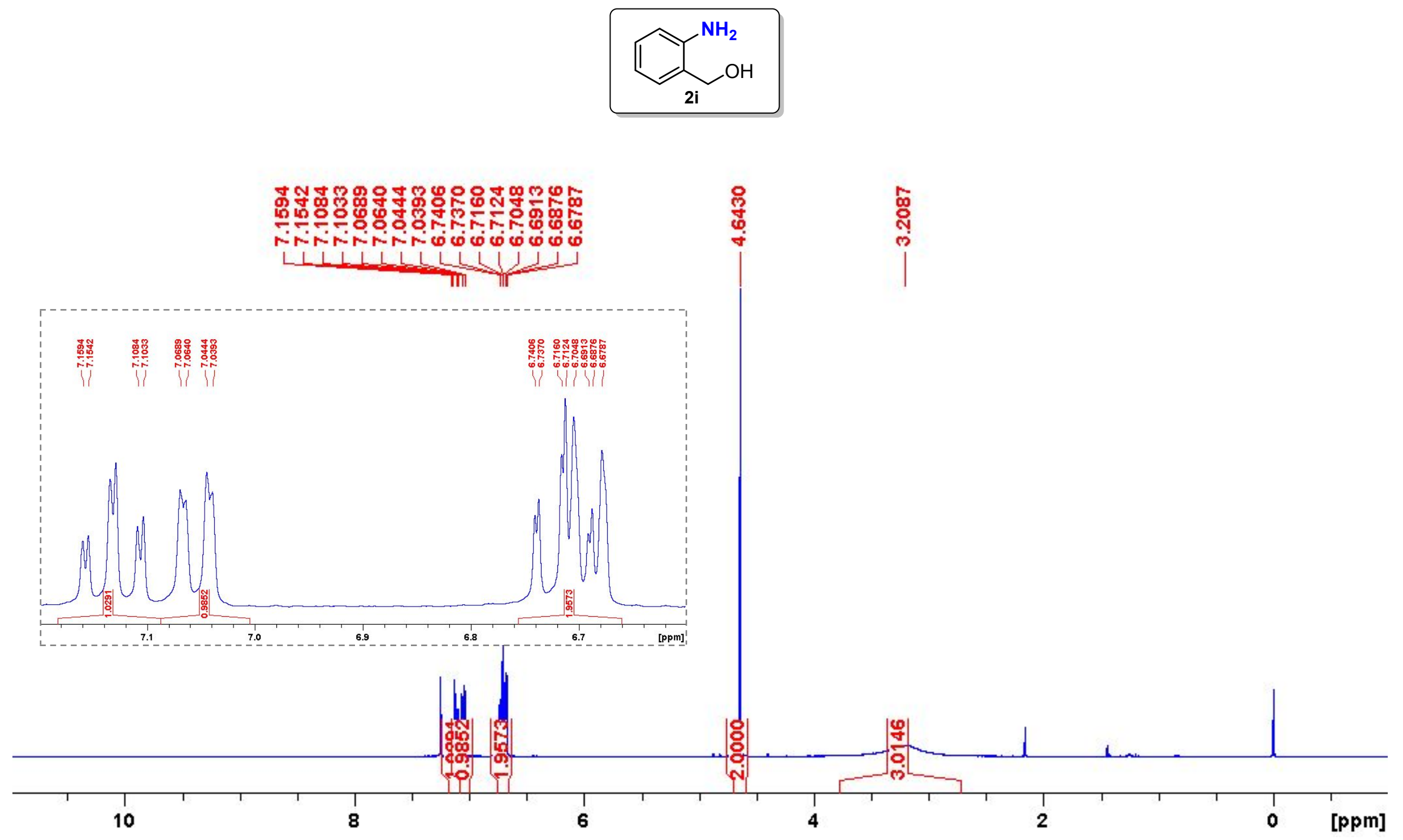

Figure S24. ${ }^{1} \mathrm{H}$ NMR spectrum $\left(300 \mathrm{MHz}, \mathrm{CDCl}_{3}\right)$ of compound $\mathbf{2 i}$. 

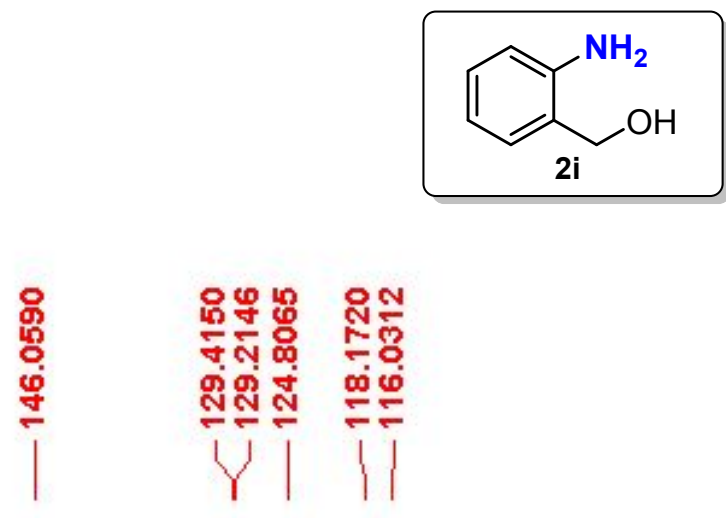

Figure S25. ${ }^{13} \mathrm{C}$ NMR spectrum $\left(75.5 \mathrm{MHz}, \mathrm{CDCl}_{3}\right)$ of compound $2 \mathbf{i}$. 